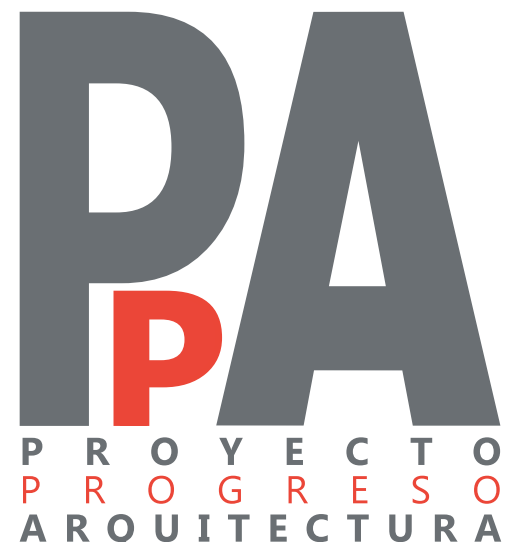

ARQUITECTURAS AMPLIADAS

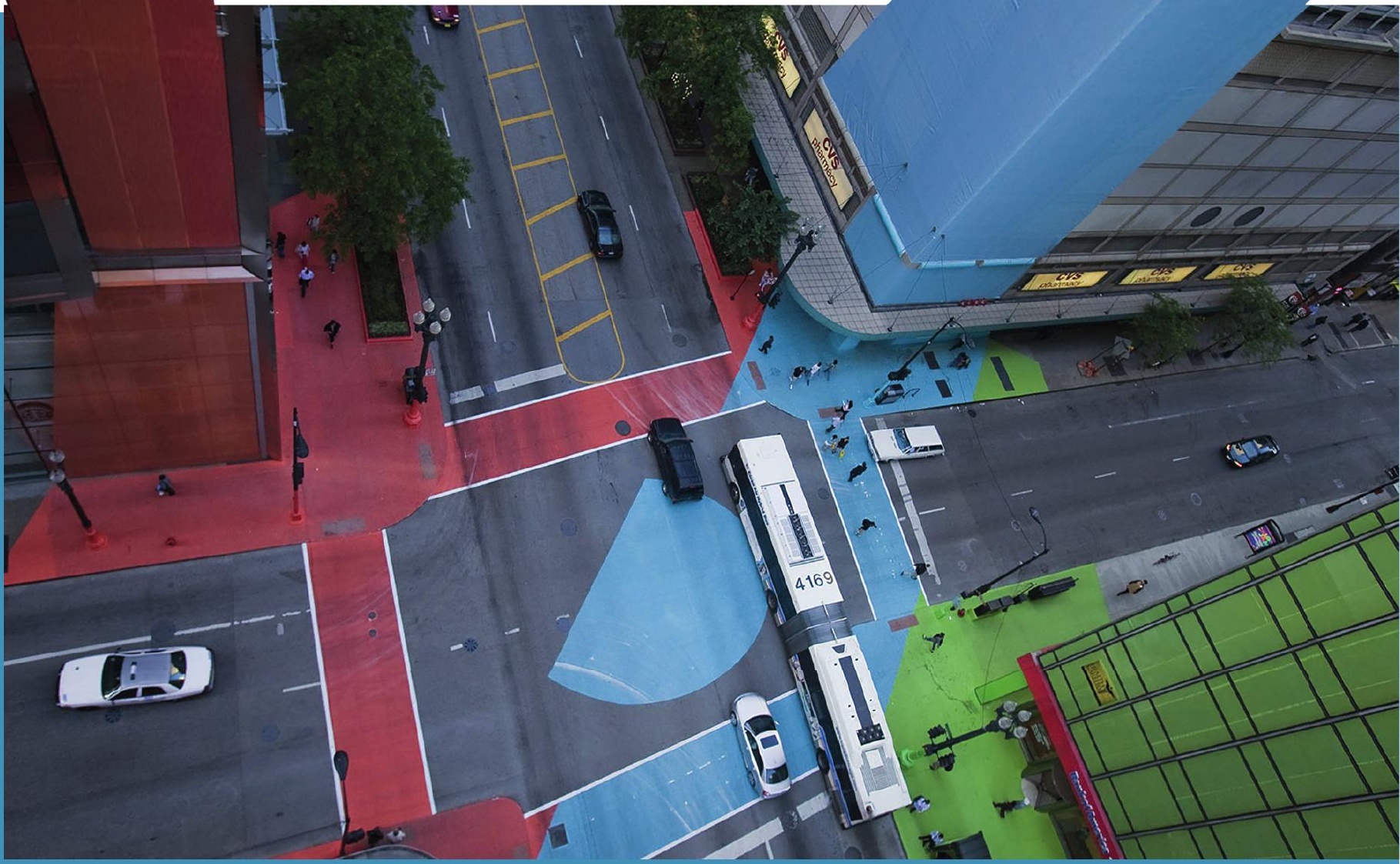




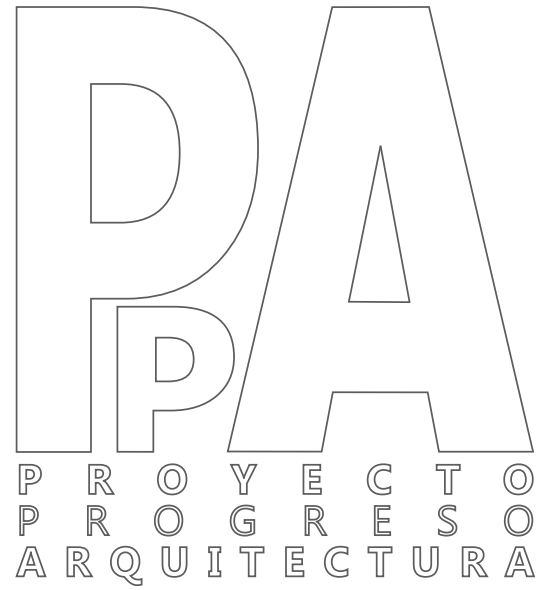

\section{ARQUITECTURAS AMPLIADA}

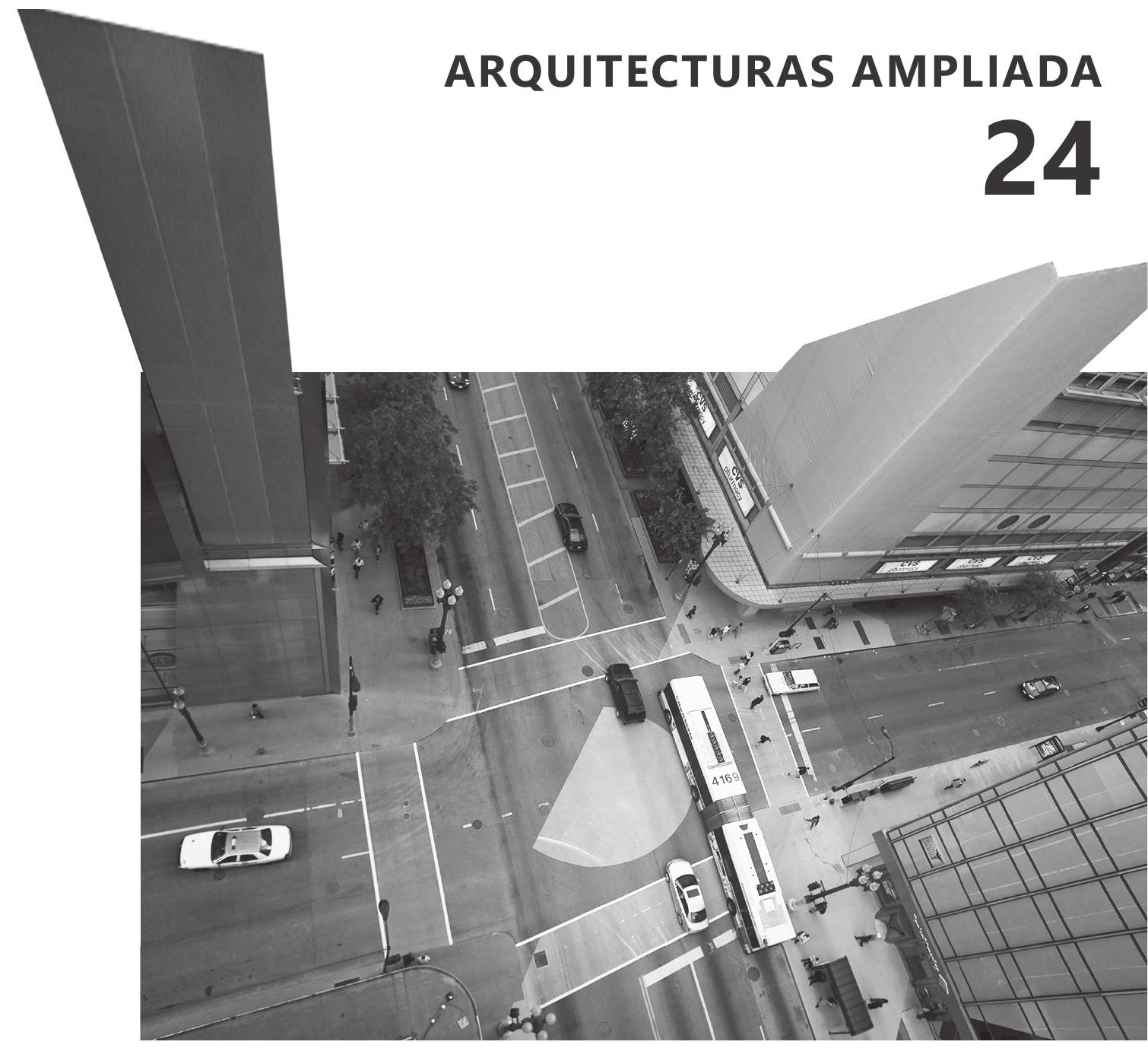

EDITORIAL UNIVERSIDAD DE SEVILLA AÑO 2019. ISSN 2171-6897 ISSNe 2173-1616 DOI: http://dx.doi.org/10.12795/ppa 


\section{REVISTA PROYECTO PROGRESO ARQUITECTURA}

N2/4

arquitecturas ampliadas
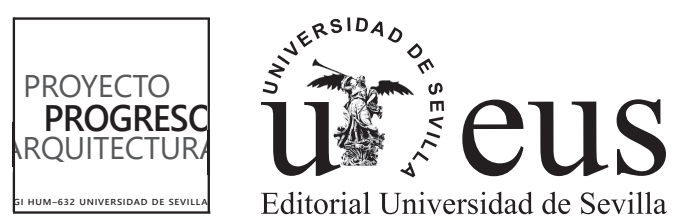


\section{arquitecturas ampliadas}

EDITA

Editorial Universidad de Sevilla. Sevilla

DIRECCIÓN CORRESPONDENCIA CIENTÍFICA

E.T.S. de Arquitectura. Avda Reina Mercedes, nº $241012-$

Sevilla.

Amadeo Ramos Carranza, Dpto. Proyectos Arquitectónicos.

e-mail: revistappa.direccion@gmail.com

\section{EDICIÓN ON-LINE}

Portal informático https://revistascientificas.us.es/index.php/ppa Portal informático Grupo de Investigación HUM-632

http://www. proyectoprogresoarquitectura.com

Portal informático Editorial Universidad de Sevilla http://www. editorial.us.es/

(c) EDITORIAL UNIVERSIDAD DE SEVILLA, 2019.

Calle Porvenir, 27. 41013 SEVILLA. Tfs. 954487447 / 954487451

Fax 954487443. [eus4@us.es] [http://www.editorial.us.es]

(c) TEXTOS: SUS AUTORES,

(C) IMÁGENES: SUS AUTORES Y/O INSTITUCIONES

DISEÑO PORTADA:

Rosa María Añón Abajas - Amadeo Ramos Carranza

En base a la fotografía: (C) Jessica Stockholder, cortesía de la artista y de Mitchell-Innes \& Nash, Nueva York

\section{DISEÑO PLANTILLA PORTADA-CONTRAPORTADA}

Miguel Ángel de la Cova Morillo-Velarde

DISEÑO PLANTILLA MAQUETACIÓN

Maripi Rodríguez

MAQUETACIÓN

Referencias Cruzadas

CORRECCION ORTOTIPOGRÁFICA

José Antonio Duarte

ISSN (ed. impresa): 2171-6897

ISSN-e (ed. electrónica): 2173-1616

DOI: http://dx.doi.org/10.12795/ppa

DEPÓSITO LEGAL: SE-2773-2010

PERIOCIDAD DE LA REVISTA: MAYO Y NOVIEMBRE

IMPRIME: PODIPRINT

Reservados todos los derechos. Ni la totalidad ni parte de esta revista puede reproducirse o transmitirse por ningún procedimiento electrónico o mecánico, incluyendo fotocopia, grabación magnética o cualquier almacenamiento de información y sistema de recuperación, sin permiso escrito de la Editorial Universidad de Sevilla.

Las opiniones y los criterios vertidos por los autores en los artículos firmados son responsabilidad exclusiva de los mismos. 


\section{DIRECCIÓN}

Dr. Amadeo Ramos Carranza. Escuela Técnica Superior de Arquitectura. Universidad de Sevilla. España

\section{SECRETARÍA}

Dra. Rosa María Añón Abajas. Escuela Técnica Superior de Arquitectura. Universidad de Sevilla. España

\section{EQUIPO EDITORIAL \\ Edición:}

Dr. Amadeo Ramos Carranza. Escuela Técnica Superior de Arquitectura. Universidad de Sevilla. España.

Dra. Rosa María Añón Abajas. Escuela Técnica Superior de Arquitectura. Universidad de Sevilla. España.

Dr. Francisco Javier Montero Fernández. Escuela Técnica Superior de Arquitectura. Universidad de Sevilla. España.

Dra. Esther Mayoral Campa. Escuela Técnica Superior de Arquitectura. Universidad de Sevilla. España.

Dr. Miguel Ángel de la Cova Morillo-Velarde. Escuela Técnica Superior de Arquitectura. Universidad de Sevilla. España.

Dr. Germán López Mena. Escuela Técnica Superior de Arquitectura. Universidad de Sevilla. España.

Dra. Gloria Rivero Lamela. Escuela Técnica Superior de Arquitectura. Universidad de Sevilla. España.

Juan José López de la Cruz. Escuela Técnica Superior de Arquitectura. Universidad de Sevilla. España.

Guillermo Pavón Torrejón. Escuela Técnica Superior de Arquitectura. Universidad de Sevilla. España.

Externos edición (asesores):

Dr. José Altés Bustelo. Escuela Técnica Superior de Arquitectura. Universidad de Valladolid. España.

Dr. Carlos Arturo Bell Lemus. Facultad de Arquitectura. Universidad del Atlántico. Colombia.

Dr. José de Coca Leicher. Escuela Técnica Superior de Arquitectura. Universidad Politécnica de Madrid. España. Dra. Patricia de Diego Ruiz. Escuela Técnica Supeiror de Arquitectura y Geodesia. Universidad Alcalá de Heranes. España.

Dr. Alfonso del Pozo y Barajas. Escuela Técnica Superior de Arquitectura. Universidad de Sevilla. España.

Dr. Jaume J. Ferrer Fores. Escola Tècnica Superior

d'Arquitectura de Barcelona. Universitat Politècnica de Catalunya. España.

Dra. Laura MArtínez Guereñu. El School of Architecture \& Design, IE University, Madrid; Segovia. España.

Dra. Clara Mejía Vallejo. Escuela Técnica Superior de Arquitectura. Universidad Politécnica de Valencia. España.

Dra. Luz Paz Agras. Escuela Técnica Superior de Arquitectura. Universidade da Coruña. España.

Dra. Marta Sequeira. CIAUD, Faculdade de Arquitectura da Universidade de Lisboa, Portugal.

\section{SECRETARÍA TÉCNICA}

Dra. Gloria Rivero Lamela. Escuela Técnica Superior de Arquitectura. Universidad de Sevilla. España.

\section{EDITORA Y COORDINACION CONTENIDOS CIENTÍFICOS DEL NÚMERO}

Dra. Rosa María Añón Abajas. Escuela Técnica Superior de Arquitectura. Universidad de Sevilla. España.

\section{COMITÉ CIÉNTIFICO}

Dr. Carlo Azteni. DICAAR. Dipartimento di Ingegneria Civile, Ambientale e Architettura. University Of Cagliari. Italia.

Dra. Maristella Casciato. GETTY Research Institute, GETTY, Los Angeles. Estados Unidos.

Dra. Anne Marie Châtelet. École Nationale Supérieure D'Architecture de Strasbourg (ENSAS). Francia.

Dr. Jean Louis Cohen. Institute of Fine Arts, New York University. Estados Unidos.

Dra. Josefina González Cubero. Escuela Técnica Superior de Arquitectura. Universidad de Valladolid. España.

Dr. José Manuel López Peláez. Escuela Técnica Superior de Arquitectura. Universidad Politécnica de Madrid. España.

Dra. Maite Méndez Baiges. Departamento de Historia del Arte. Universidad de Málaga. España.

Dr. Dietrich C. Neumann. Brown University In Providence, Ri (John Nicholas Brown Center For Public Humanities And Cultural Heritage). Estados Unidos.

Dr. Víctor Pérez Escolano. Catedrático Historia, Teoría y Composición Arquitectónicas. Escuela Técnica Superior de Arquitectura. Universidad de Sevilla. España.

Dr. Jorge Torres Cueco. Catedrático Proyectos Arquitectónicos. Escuela Técnica Superior de Arquitectura. Universitat Politècnica de València. España.

Dr. ir. Frank van der Hoeven, TU DELFT. Architecture and the Built Environment, Netherlands

\section{CORRESPONSALES}

Pablo de Sola Montiel. The Berlage Centre for Advanced Studies in Architecture and Urban Design. Paises Bajos.

Dr. Plácido González Martínez. Tongji University Caup (College Of architectura \& Urban Planing). Shangai, China.

Patrícia Marins Farias. Faculdade de Arquitetura. Universidade Federal da Bahia. Brasil.

Dr. Daniel Movilla Vega. Umeå School of Architecture. Umeå University. Suecia.

Dr. Pablo Sendra Fernández. The Bartlett School of Planning. University College London. Inglaterra.

Alba Zarza Arribas. Centro de Estudos Arnaldo Araújo, Porto. Portugal.

Dra. María Elena Torres Pérez. Facultad de Arquitectura. Universidad Autónoma de Yucatán, Mérida. México.

\section{TEXTOS VIVOS}

Juan José López de la Cruz. Escuela Técnica Superior de Arquitectura. Universidad de Sevilla. España.

Dr. Francisco Javier Montero Fernández. Escuela Técnica Superior de Arquitectura. Universidad de Sevilla. España. Dra. Esther Mayoral Campa. Escuela Técnica Superior de Arquitectura. Universidad de Sevilla. España. 


\section{SERVICIOS DE INFORMACIÓN}

\section{CALIDAD EDITORIAL}

La Editorial Universidad de Sevilla cumple los criterios establecidos por la Comisión Nacional Evaluadora de la Actividad Investigadora para que lo publicado por el mismo sea reconocido como "de impacto" (Ministerio de Ciencia e Innovación, Resolución 18939 de 11 de noviembre de 2008 de la Presidencia de la CNEAI, Apéndice I, BOE n² 282, de 22.11.08).

La Editorial Universidad de Sevilla forma parte de la U.N.E. (Unión de Editoriales Universitarias Españolas) ajustándose al sistema de control de calidad que garantiza el prestigio e internacionalidad de sus publicaciones.

PUBLICATION QUALITY

The Editorial Universidad de Sevilla fulfils the criteria established by the National Commission for the Evaluation of Research Activity (CNEAI) so that its publications are recognised as "of impact" (Ministry of Science and Innovation, Resolution 18939 of 11 November 2008 on the Presidency of the CNEAl, Appendix I, BOE No 282, of 22.11.08).

The Editorial Universidad de Sevilla operates a quality control system which ensures the prestige and international nature of its publications, and is a member of the U.N.E. (Unión de Editoriales Universitarias Españolas-Union of Spanish University Publishers).

Los contenidos de la revista PROYECTO, PROGRESO, ARQUITECTURA aparecen en:

bases de datos: indexación
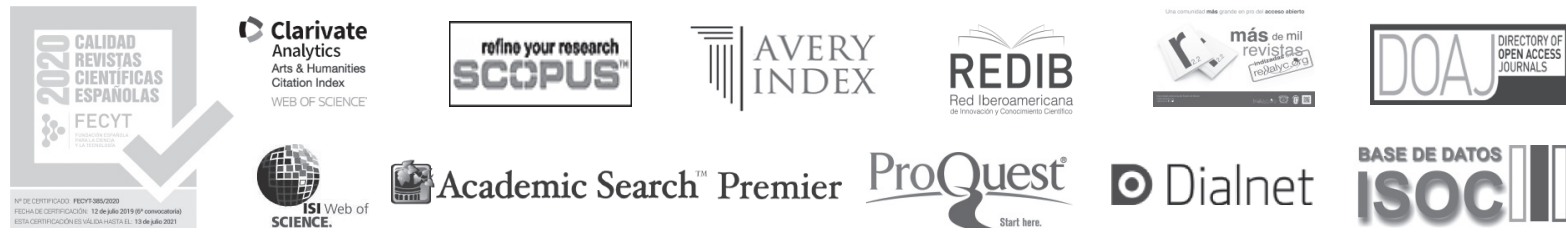

D Dialnet

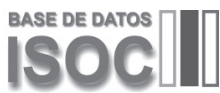

SELLO DE CALIDAD EDITORIAL FECYT 2019. RENOVADO 2020. (Cuartil C3)

WoS. Arts \& Humanities Citation Index

WoS. ESCl - Emerging Sources Citation Index

SCOPUS

AVERY. Avery Index to Architectural Periodicals

REBID. Red Iberoamericana de Innovación y Conocimiento Científico

REDALYC. Red de Revistas Científicas de América Latina y el Caribe, España y Portugal.

EBSCO. Fuente Académica Premier

EBSCO. Art Source

DOAJ, Directory of Open Access Journals

PROQUEST (Arts \& Humanities, full text)

DIALNET

ISOC (Producida por el CCHS del CSIC)

DRIJ. Directory of Research Journals Indexing

SJR (2019): 0.100, H index: 2

\section{catalogaciones: criterios de calidad}

RESH (Revistas Españolas de Ciencias Sociales y Humanidades).

Catálogos CNEAI (16 criterios de 19). ANECA (18 criterios de 21). LATINDEX (35 criterios sobre 36).

DICE (CCHS del CSIC, ANECA).

MIAR, Matriu d'Informació per a l'Avaluació de Revistes. IDCS 2018: 10,500. Campo ARQUITECTURA

CLASIFICACIÓN INTEGRADA DE REVISTAS CIENTÍFICAS (CIRC-CSIC): A

ERIHPLUS

SCIRUS, for Scientific Information.

ULRICH'S WEB, Global Serials Directory.

ACTUALIDAD IBEROAMERICANA.

\section{catálogos on-line bibliotecas notables de arquitectura:}

CLIO. Catálogo on-line. Columbia University. New York

HOLLIS. Catálogo on-line. Harvard University. Cambridge. MA

SBD. Sistema Bibliotecario e Documentale. Instituto Universitario di Architettura di Venezia

OPAC. Servizi Bibliotecari di Ateneo. Biblioteca Centrale. Politecnico di Milano

COPAC. Catálogo colectivo (Reino Unido)

SUDOC. Catálogo colectivo (Francia)

ZBD. Catálogo colectivo (Alemania)

REBIUN. Catálogo colectivo (España)

OCLC. WorldCat (Mundial) 
EVALUACIÓN EXTERNA POR PARES Y ANÓNIMA.

El Consejo Editorial remitirá el artículo a dos expertos revisores anónimos dentro del campo específico de investigación y crítica de arquitectura, según el modelo doble ciego.

El director de la revista comunicará a los autores el resultado motivado de la evaluación por correo electrónico, en la dirección que éstos hayan utilizado para enviar el artículo. El director comunicará al autor principal el resultado de la revisión (publicación sin cambios; publicación con correcciones menores; publicación con correcciones importantes; no aconsejable para su publicación), así como las observaciones y comentarios de los revisores.

Si el manuscrito ha sido aceptado con modificaciones, los autores deberán reenviar una nueva versión del artículo, atendiendo a las demandas y sugerencias de los evaluadores externos. Los artículos con correcciones importantes serán remitidos al Consejo Asesor para verificar la validez de las modificaciones efectuadas por el autor. Los autores pueden aportar también una carta al Consejo Editorial en la que indicarán el contenido de las modificaciones del artículo. Los artículos con correcciones importantes serán remitidos al Consejo Asesor para verificar la validez de las modificaciones efectuadas por el autor.

\section{DECLARACIÓN ÉTICA SOBRE PUBLICACIÓN Y MALAS PRÁCTICAS}

La revista PROYECTO, PROGRESO, ARQUITECTURA (PPA) está comprometida con la comunidad académica en garantizar la ética y calidad de los artículos publicados. Nuestra revista tiene como referencia el Código de Conducta y Buenas Prácticas que, para editores de revistas científicas, define el COMITÉ DE ÉTICA DE PUBLICACIONES (COPE).

Así nuestra revista garantiza la adecuada respuesta a las necesidades de los lectores y autores, asegurando la calidad de lo publicado, protegiendo y respetando el contenido de los artículos y la integridad de los mismo. El Consejo Editorial se compromete a publicar las correcciones, aclaraciones, retracciones y disculpas cuando sea preciso.

En cumplimiento de estas buenas prácticas, la revista PPA tiene publicado el sistema de arbitraje que sigue para la selección de artículos así como los criterios de evaluación que deben aplicar los evaluadores externos -anónimos y por pares, ajenos al Consejo Editorial-. La revista PPA mantiene actualizados estos criterios, basados exclusivamente en la relevancia científica del artículo, originalidad, claridad y pertinencia del trabajo presentado.

Nuestra revista garantiza en todo momento la condifencialidad del proceso de evaluación: el anonimato de los evaluadores y de los autores; el contenido evaluado; los informes razonados emitidos por los evaluadores y cualquier otra comunicación emitida por los consejos Editorial, Asesor y Científico si así procediese.

Igualmente quedan afectados de la máxima confidencialidad las posibles aclaraciones, reclamaciones o quejas que un autor desee remitir a los comités de la revista o a los evaluadores del artículo.

La revista PROYECTO, PROGRESO, ARQUITECTURA (PPA) declara su compromiso por el respeto e integridad de los trabajos ya publicados. Por esta razón, el plagio está estrictamente prohibido y los textos que se identifiquen como plagio o su contenido sea fraudulento, serán eliminados o no publicados por la revista PPA. La revista actuará en estos casos con la mayor celeridad posible. Al aceptar los términos y acuerdos expresados por nuestra revista, los autores han de garantizar que el artículo y los materiales asociados a él son originales o no infringen derechos de autor. También los autores tienen que justificar que, en caso de una autoría compartida, hubo un consenso pleno de todos los autores afectados y que no ha sido presentado ni publicado con anterioridad en otro medio de difusión.

\section{EXTERNAL ANONYMOUS PEER REVIEW.}

Editorial Board will be sent to two anonymous experts, within the specific field of architectural investigation and critique, for a double blind review.

The Director of the journal will communicate the result of the reviewers evaluations to the authors by electronic mail, to the address used to send the article. The Director will communicate the result of the review (publication without changes; publication with minor corrections; publication with significant corrections; its publication is not advisable), as well as the observations and comments of the reviewers, to the main author.

If the manuscript has been accepted with modifications, the authors will have to resubmit a new version of the article, addressing the requirements and suggestions of the external reviewers. The articles with corrections will be sent to Advisory Board for verification of the validity of the modifications made by the author. The authors can also send a letter to the Editorial Board, in which they will indicate the content of the modifications of the article.

\section{ETHICS STATEMENT ON PUBLICATION AND BAD PRACTICES} PROYECTO, PROGRESO ARQUITECTURA (PPA) makes a commitment to the academic community by ensuring the ethics and quality of its published articles. As a benchmark, our journal uses the Code of Conduct and Good Practices which, for scientific journals, is defined for editors by the PUBLICATION ETHICS COMMITTEE (COPE).

Our journal thereby guarantees an appropriate response to the needs of readers and authors, ensuring the quality of the published work, protecting and respecting the content and integrity of the articles. The Editorial Board will publish corrections, clarifications, retractions and apologies when necessary.

In compliance with these best practices, PPA has published the arbitration system that is followed for the selection of articles as well as the evaluation criteria to be applied by the anonymous, external peer-reviewers. PPA keeps these criteria current, based solely on the scientific importance, the originality, clarity and relevance of the presented article.

Our journal guarantees the confidentiality of the evaluation process at all times: the anonymity of the reviewers and authors; the reviewed content; the reasoned report issued by the reviewers and any other communication issued by the editorial, advisory and scientific boards as required.

Equally, the strictest confidentiality applies to possible clarifications, claims or complaints that an author may wish to refer to the journal's committees or the article reviewers.

PROYECTO, PROGRESO ARQUITECTURA (PPA) declares its commitment to the respect and integrity of work already published. For this reason, plagiarism is strictly prohibited and texts that are identified as being plagiarized, or having fraudulent content, will be eliminated or not published in PPA. The journal will act as quickly as possible in such cases. In accepting the terms and conditions expressed by our journal, authors must guarantee that the article and the materials associated with it are original and do not infringe copyright. The authors will also have to warrant that, in the case of joint authorship, there has been full consensus of all authors concerned and that the article has not been submitted to, or previously published in, any other media. 
EVALUADORES EXTERNOS (publicación cada cuatro números, dos años). NÚMEROS 21 a 24 (incluidos)

Álvarez Álvarez, Darío. Catedrático de Universidad / Departamento de Teoría de la Arquitectura y Proyectos Arquitectónicos / ETS Arquitectura / Universidad de Valladolid / España.

Arrieta Berdasco, Valentín. Doctor Arquitecto, Profesor Asociado / Departamento de Teoría de la Arquitectura y Proyectos Arquitectónicos / ETS Arquitectura / Universidad de Valladolid / España.

Bardí i Milá, Berta. Profesora Asociada doctora / / Departamento de Projectes Arquitectònics / ETS Arquitectura / Universitat Politècnica de Catalunya, Barcelona Tech / España.

Bergera Serrano, Iñaki. Titular de Universidad / Unidad Predepartamental de Arquitectura / Área Proyectos Arquitectónicos / El y Arquitectura / Universidad de Zaragoza / España.

Bobbink, Inge. Dr. ir. Architecture / Section of landscape architecture / TU Delft / Países Bajos.

Burriel Bielza, Luis. Profesor Titular / École Nationale Supérieure d'Architecture de Paris-Belleville / Francia

Calatrava Escobar, Juan. Catedrático de Universidad / Departamento de Construcciones Arquitectónicas / ETS Arquitectura / Universidad de Granada / España.

Castellanos Gómez, Raúl. Titular de Universidad / Departamento de Proyectos Arquitectónicos / ETS Arquitectura / Universidad Politécnica de Valencia / España.

Centellas Soler, Miguel. Titular de Universidad / Departamento de Arquitectura y Tecnología de la Edificación / ETS Arquitectura y Edificación / Universidad Politécnica de Cartagena / España.

Chías Navarro, Pilar. Catedrática de Universidad / Departamento de Arquitectura / ETS Arquitectura y Geodesia / Universidad de Alcalá de Henares / España.

de Diego Ruiz, Patricia. Doctora arquitecta, Profesora Asociada / Departamento de Arquitectura / ETS Arquitectura y Geodesia / Universidad de Alcalá de Henares / España.

de la Iglesia Salgado, Félix. Profesor Contratado Doctor / Departamento de Proyectos Arquitectónicos / ETS Arquitectura / Universidad de Sevilla / España.

de la O Cabrera, Manuel Rodrigo. Doctor Arquitecto, Profesor Asociado / Departamento de Composición Arquitectónica / ETS Arquitectura / Universidad Politécnica de Madrid / España.

Delgado Orusco, Eduardo. Profesor Ayudante Doctor / Departamento de Arquitectura. Área de Proyectos Arquitectónicos / Escuela de Ingeniería y Arquitectura / Universidad de Zaragoza / España.

Deltell Pastor, Juan. Titular de Universidad / Departamento de Proyectos Arquitectónicos / ETS Arquitectura / Universitat Politècnica de València / España.

Diañez Rubio, Pablo. Titular de Universidad / Departamento de Proyectos Arquitectónicos / ETS Arquitectura / Universidad de Sevilla / España.

Díaz Segura, Alfonso. Titular de Universidad / Proyectos, Teoría y Técnica del Diseño y la Arquitectura / ETS Arquitectura / Universidad CEU Cardenal Herrera, Valencia / España.

Domingo Calabuig, Débora. Titular de Universidad / Departamento de Proyectos Arquitectónicos / ETS Arquitectura / Universidad Politécnica de Valencia / España.

Fernández Fariña, Almudena. Profesora Contratada Doctora / Departamento de Pintura / Facultad de Bellas Artes / Universidad de Vigo / España.

Fernández-Trapa de Isasi, Justo. Catedrático de Universidad / Departamento de Proyectos Arquitectónicos / ETS Arquitectura / Universidad Politécnica de Madrid / España.

García Escudero, Daniel. Dr. Arquitecto, Profesor Lector / Departamento de Projectes Arquitectònics / ETS Arquitectura / Universitat Politècnica de Catalunya, Barcelona Tech / España.

González Cubero, Josefina. Titular de Universidad / Departamento de Teoría de la Arquitectura y Proyectos Arquitectónicos / ETS Arquitectura / Universidad de Valladolid / España.

González Fraile, Eduardo. Catedrático de Universidad / Departamento de Teoría de la Arquitectura y Proyectos Arquitectónicos / ETS Arquitectura / Universidad de Valladolid / España.

Gorostiza López, Jorge. Doctor Arquitecto. Cineasta / España.

Hernández Moreno, Silverio. Profesor investigador titular / Facultad de Arquitectura y Diseño / Universidad Autónoma del Estado de México / México.

Labarta Aizpún, Carlos. Titular de Universidad / Unidad Predepartamental de Arquitectura / Área Proyectos Arquitectónicos / Escuela de Ingeniería y Arquitectura / Universidad de Zaragoza / España.

Lizondo Sevilla, Laura. Titular de Universidad / Departamento de Proyectos Arquitectónicos / ETS Arquitectura / Universidad Politécnica de Valencia / España.

Llopis Verdú, Jorge. Catedrático de Universidad / Departamento de Expresión Gráfica Arquitectónica / ETS Arquitectura / Universidad Politécnica de Valencia / España.

López Bahut, Emma. Profesora Contratada Doctora / Departamento de Proyectos arquitectónicos, Urbanismo y Composición / ETS Arquitectura / Universidade da Coruña / España.

López Fernández, Andrés. Titular de Universidad / Departamento de Proyectos Arquitectónicos / ETS Arquitectura / Universidad de Sevilla / España.

Loren Méndez, Mar. Catedrática de Universidad / Departamento de Historia, Teoría y Composición Arquitectónica / ETS Arquitectura / Universidad de Sevilla / España.

Maino Ansaldo, Sandro. Doctor Arquitecto / Departamento Arquitectura / Universidad Técnica Federico Santa María / Chile.

Mària i Serrano, Magda. Profesora Contratada Doctor / Departamento de Projectes Arquitectònics / ETS Arquitectura del Vallès / Universitat Politècnica de Catalunya / España

Marson, Anna. Profesora Ordinario / Dipartimento di culture del progetto / Istituto Universitario di Architettura di Venezia / Università di Venezia / Italia.

Martínez Díaz, Ángel. Titular de Universidad / Departamento de Ideación Gráfica Arquitectónica / ETS Arquitectura / Universidad Politécnica de Madrid / España. 
Mejía Vallejo, Clara. Titular de Universidad / Departamento de Proyectos Arquitectónicos / ETS Arquitectura / Universidad Politécnica de Valencia / España.

Mercader Moyano, Pilar. Titular de Universidad / Departamento de Proyectos Arquitectónicos / ETS Arquitectura / Universidad de Sevilla / España.

Mercé Hospital, José María. Catedrático de Universidad / Departamento de Proyectos Arquitectónicos / ETS Arquitectura y Geodesia / Universidad Alcalá de Henares / España.

Merí de la Maza, Ricardo. Titular de Universidad / Departamento de Proyectos Arquitectónicos / ETS Arquitectura / Universidad Politécnica de Valencia / España.

Mestre Martínez, Nieves. Doctora arquitecta, Profesora Asociada / Departamento de Proyectos Arquitectónicos / ETS Arquitectura / Universidad Politécnica de Madrid / España.

Millán Gómez, Antonio. Catedrático de Universidad / Departamento d’Expressió Gràfica Arquitectònica I / ETS Arquitectura del Vallès / Universitat Politècnica de Catalunya / España.

Moreno Pérez, José Ramón. Titular de Universidad / Dpto. Historia, Teoría y Composición Arquitectónica / ETS Arquitectura / Universidad de Sevilla / España.

Nijhuis, Steffen. Associate Professor. Head of Landscape Architecture Research / Section of Landscape Architecture / Department of Urbanism / Faculty of Architecture and the Built Environment / TU Delft / Países Bajos.

Ojeda Rivera, Juan Francisco. Catedrático de Universidad / Departamento de Geografía, Historia y Filosofía / Universidad Pablo de Olavide, Sevilla / España.

Paz-Agras, Luz. Profesora Ayudante doctor / Departamento de Proyectos Arquitectónicos, Urbanismo y Composición. Área de Composición Arquitectónica / ETS Arquitectura / Universidad de A Coruña / España

Pérez Moreno, Lucía C. Titular de Universidad / Departamento de Arquitectura. Área de Composición Arquitectónica / Escuela de Ingeniería y Arquitectura / Universidad de Zaragoza / España.

Pina Lupiañez, Rafael. Profesor Contratado Doctor / Departamento de Proyectos Arquitectónicos / ETS Arquitectura / Universidad Politécnica de Madrid / España

Oliverira do Nascimento, Francisco. Professor Auxiliar / Faculdade de Arquitetura / Universidade de Lisboa / Portugal.

Rovira Llobera, Teresa. Titular de Universidad / Departamento de Projectes Arquitectònics / ETS Arquitectura / Universitat Politècnica de Catalunya, Barcelona Tech / España.

Ruiz Rosa, José Antonio. Catedrático de Universidad / Departamento de Expresión Gráfica Arquitectónica / ETS Arquitectura / Universidad de Sevilla. / España.

Sabaté Bel, Joaquín. Catedrático de Universidad / Departamento de Urbanismo y Ordenación del Territorio / ETS Arquitectura / Universitat Politècnica de Catalunya, Barcelona Tech / España

Sainz Gutiérrez Victoriano. Titular de Universidad / Departamento de Urbanismo y Ordenación del Territorio / ETS Arquitectura / Universidad de Sevilla / España

Sánchez Lampreave, Ricardo. Profesor Titular / Área de Composición Arquitectónica / Escuela de Ingeniería y Arquitectura / Universidad de Zaragoza / España.

Sambricio R. Echegaray, Carlos. Catedrático de Universidad / Departamento de Composición Arquitectónica / ETS Arquitectura / Universidad Politécnica de Madrid / España. / España.

Santamarina-Macho, Carlos. España. Doctor Arquitecto, Profesor Asociado / Departamento de Teoría de la Arquitectura y Proyectos Arquitectónicos / ETS Arquitectura / Universidad de Valladolid / España

Senra Fernández-Miranda, Ignacio. Doctor Arquitecto, Profesor Asociado / Departamento de Proyectos Arquitectónicos / ETS Arquitectura / Universidad Politécnica de Madrid / España.

Sentieri Omarrementeria, Carla. Titular de Universidad / Departamento de Proyectos Arquitectónicos / ETS Arquitectura / Universidad Politécnica de Valencia / España.

Sequeira Marta. Professora doctora / ISCTE - Instituto Universitário de Lisboa/ Universidade Autónoma de Lisboa / CIAUD - Faculdade de Arquitectura da Universidade de Lisboa / Portugal.

Sola Alonso, José Ramón. España. Profesor Contratado Doctor / Departamento de Teoría de la Arquitectura y Proyectos Arquitectónicos / ETS Arquitectura / Universidad de Valladolid / España.

Trillo Martínez, Valentín. Doctor arquitecto Profesor Asociado / Departamento de Proyectos Arquitectónicos / ETS Arquitectura / Universidad de Sevilla / España.

Villalobos Alonso, Daniel. Titular de Universidad / Departamento de Teoría de la Arquitectura y Proyectos Arquitectónicos / ETS Arquitectura / Universidad de Valladolid / España.

Verde Zein, Ruth. Doctora arquitecto e investigadora / Facultad de Arquitectura y Urbanismo / Universidad Presbiteriana Mackenzie. São Paulo / Brasil.

\section{ESTADÍSTICAS PUBLICACIÓN (publicación cada cuatro números, dos años). NÚMEROS 1 a 20 (incluidos)}

Total artículos recibidos: 546

Total artículos publicados: 199 (36,45\%)

Total artículos rechazados: $347(63,55 \%)$

Total artículos publicados de autores pertenecientes a los diferentes consejos o comités organizadores de la revista y Grupo de Investigación "proyecto, progreso, arquitectura"(endogamia): 20 (10,05\%)

Total artículos publicados de autores pertenecientes a la Universidad de Sevilla: 53 (26,63\%)

Total artículos publicados de autores externos a los diferentes consejos o comités organizadores de la revista y Grupo de Investigación

"proyecto, progreso, arquitectura": 179 (89,95\%)

Total artículos publicados de autores extranjeros: 17 (8,54\%) 


\section{arquitecturas ampliadas}

índice

editorial

DESPEJAR LA ARQUITECTURA, LIBERAR EL ESPACIO Y AMPLIAR CONCEPTOS / UNCLUTTER ARCHITECTURE, FREE UP SPACE AND EXPAND CONCEPTS

Rosa María Añón-Abajas - (DOI: http://dx.doi.org/10.12795/ppa.2021.i24.10)

entre líneas

PAISAJES ARQUITECTÓNICOS Y MEMORIAS DE LA CIUDAD / ARCHITECTURAL LANDSCAPES AND MEMORIES OF THE CITY

Darío Álvarez Álvarez - (D0l: http://dx.doi.org/10.12795/ppa.2021.i24.01)

artículos

LINAZASORO EN REIMS. EL ESPACIO PÚBLICO COMO MEMORIA DEL LUGAR / LINAZASORO IN REIMS. THE PUBLIC SPACE AS THE MEMORY OF THE PLACE

Victoriano Sainz Gutiérrez - (DOl: http://dx.doi.org/10.12795/ppa.2021.i24.02)

EDITAR VS. CONSTRUIR: UNA ECOLOGÍA DE LO INVISIBLE. AMPLIFICAR LA COMPRENSIÓN DE LAS TÉCNICAS DE PROYECTO / EDITING VS. BUILDING: AN ECOLOGY OF THE INVISIBLE. AMPLIFYING THE UNDERSTANDING OF ARCHITECTURAL DESIGN TECHNIQUES

Paula Victoria Álvarez Benítez - (DOI: http://dx.doi.org/10.12795/ppa.2021.i24.03)

CUANDO LA PINTURA AMPLÍA LA ARQUITECTURA: INTERVENCIONES REALIZADAS EN EL ESPACIO PÚBLICO / WHEN PAINTING ENHANCES ARCHITECTURE: INTERVENTIONS IN A PUBLIC SETTING Aurora Alcaide-Ramírez; Ana Ruiz-Abellón - (D0l: http://dx.doi.org/10.12795/ppa.2021.i24.04)

UN EDIFICIO INVISIBLE. NUEVO AULARIO DE LA FACULTAD DE DERECHO DE LA UNIVERSIDAD DE ZARAGOZA (1983-1996) / AN INVISIBLE BUILDING. NEW LECTURE ROOM BUILDING OF THE FACULTY OF LAW OF THE UNIVERSITY OF ZARAGOZA (1983-1996)

Luis Miguel Lus-Arana; Lucía Carmen Pérez-Moreno - (D0l: http://dx.doi.org/10.12795/ppa.2021.i24.05)

ARQUITECTURAS AMPLIADAS. EL PABELLÓN DE EXPOSICIONES EN LA CASA DE CAMPO DE MADRID) / EXPANDED ARCHITECTURES. THE EXHIBITION PAVILION AT THE CASA DE CAMPO IN MADRID José de Coca Leicher - (DOI: http://dx.doi.org/10.12795/ppa.2021.i24.06)

reseña bibliográfica TEXTOS VIVOS

RAFAEL MONEO VALLÉS: LA VIDA DE LOS EDIFICIOS. LA MEZQUITA DE CÓRDOBA, LA LONJA DE SEVILLA Y UN CARMEN EN GRANADA

Víctor Pérez Escolano - (DOl: http://dx.doi.org/10.12795/ppa.2021.i24.07)

FRANCISCO DE GRACIA: CONSTRUIR EN LO CONSTRUIDO. LA ARQUITECTURA COMO MODIFICACIÓN

Pablo Diañez Rubio - (DOl: http://dx.doi.org/10.12795/ppa.2020.i24.08)

FRÉDÉRIC DRUOT, ANNE LACATON \& JEAN-PHILIPPE VASSAL PLUS: LA VIVIENDA COLECTIVA. TERRITORIO DE EXCEPCIÓN

Javier Terrados Cepeda - (DOI: http://dx.doi.org/10.12795/ppa.2021.i24.09) 


\title{
LINAZASORO EN REIMS. EL ESPACIO PÚBLICO COMO MEMORIA DEL LUGAR
}

\author{
LINAZASORO IN REIMS. THE PUBLIC SPACE AS THE MEMORY OF THE PLACE
}

Victoriano Sainz Gutiérrez (https://orcid.org/0000-0002-8125-5333)

RESUMEN El problema de la relación entre lo antiguo y lo nuevo recorre toda la obra construida del arquitecto español José Ignacio Linazasoro; de hecho, sus mejores proyectos son probablemente aquellos en los que ha tenido que trabajar con edificios preexistentes, estableciendo un diálogo entre memoria y contemporaneidad. Esto vale sobre todo para sus actuaciones en espacios públicos situados en entornos de carácter patrimonial: el caso de la plaza frente a la catedral de Reims resulta emblemático a este respecto. El proyecto de Linazasoro para Reims nace de una reflexión sobre el significado urbano de las catedrales góticas francesas y, a partir de ahí, recuperando las trazas del tejido medieval circundante, plantea una reordenación de la plaza. Lo singular de su proyecto es que la entiende como una arquitectura expandida que forma parte de la catedral y la reintegra en la ciudad. Con un lenguaje contemporáneo, pero esencial, cargado de resonancias históricas, juega con la topografía, los pavimentos y la vegetación para recuperar la memoria del lugar. El artículo se ocupa también de contextualizar la intervención en la obra del arquitecto donostiarra y en los debates franceses sobre el modo de tratar este tipo de espacios.

PALABRAS CLAVE memoria; contemporaneidad; espacio público; catedral gótica; José Ignacio Linazasoro; Reims

SUMMARY The problem of the relationship between the old and the new runs through the entire built work of Spanish architect José Ignacio Linazasoro. In fact, his best projects are probably those in which he works with pre-existing buildings, establishing a dialogue between memory and contemporaneity. This is especially true of his interventions on public spaces in heritage surroundings, and the case of the square in front of Reims Cathedral $(1992,2003-2008)$ is emblematic in this respect. Linazasoro's project for Reims starts from a reflection on the urban meaning of French Gothic cathedrals and from there he considers restructuring the square by recovering the outlines of the surrounding medieval fabric. One of the unique aspects of his conceptual design is that he sees the square as expanded architecture: the plaza becomes part of the cathedral and reintegrates it into the city. With a contemporary but essential language brimming with historical resonances, he plays with the topography, paving and vegetation to recover the memory of the place. The article also places the intervention of the Basque architect in the context of his own work and of the debate in France on the way to treat this type of spaces.

KEYWORDS memory; contemporaneity; public space; Gothic cathedral; José lgnacio Linazasoro; Reims

Persona de contacto / Corresponding author: vsainz@us.es. Escuela Técnica Superior de Arquitectura. Universidad de Sevilla. España. 
$\mathrm{E}$ ntre los edificios que históricamente han experimentado más transformaciones para adaptar sus espacios a nuevas exigencias se encuentran las catedrales. Son construcciones que, a menudo, se han ido conformando a lo largo del tiempo, con una vida rica y compleja que ha permitido que lleguen hasta nosotros ejemplos insignes de la arquitectura antigua, integrados en edificios más recientes: "Si la arquitectura se estableció con firmeza -ha escrito Moneo a este respecto-, permanecerá abierta a nuevas intervenciones que prolongarán indefinidamente la vida del edificio"1. Podemos pensar en el complejo organismo de la catedral de Canterbury, cuyo proceso de construcción se prolongó durante casi diez siglos, por mencionar solo un ejemplo.

Pero hoy sabemos que esos grandes conjuntos monumentales son inseparables del entorno urbano donde se insertan, el cual de algún modo los completa, al permitir que sean percibidos de una manera determinada. Así, la vida de los edificios se halla profundamente ligada a la de la ciudad que los alberga y, en el caso de las catedrales, de manera particular a los espacios públicos que las rodean, por cuanto estos han servido de soporte para numerosas actividades estrechamente vinculadas a su uso desde tiempos remotos. Y es que "a la catedral no se entraba solamente para orar; allí se reunían las asociaciones de los oficios y toda la comuna para sus reuniones civiles. [...] Los hombres de negocios consideraban, pues, este monumento como propio"2.

Por eso, supuesta esa relación orgánica entre el espacio interior del templo y el espacio urbano circundante, cuando nos referimos a las experiencias de transformación realizadas sobre las catedrales históricas no debemos olvidar las intervenciones que se han llevado a cabo en sus inmediaciones, pues de ellas depende con frecuencia que se haya conseguido una adecuada integración del edificio en su entorno, máxime cuando muchas de las operaciones llevadas a cabo en épocas recientes han tendido a aislar el monumento, independizándolo del 


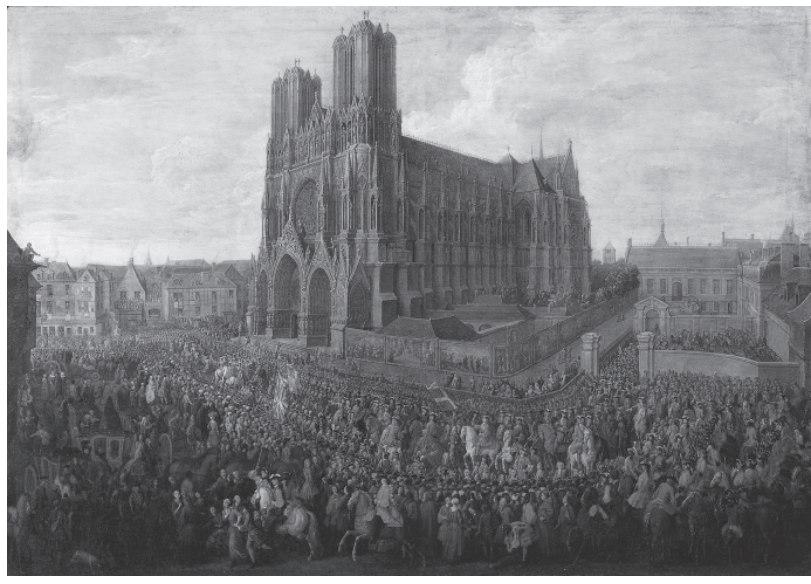

1

tejido urbano del que formaba parte ${ }^{3}$. En este sentido, la intervención en el espacio público que rodea a las catedrales se convierte en un elemento crucial de cualquier proyecto de modificación y ampliación del edificio que pretenda aportarle nuevos valores arquitectónicos. A tratar de ilustrarlo está dedicado el presente artículo.

\section{J. I. LINAZASORO Y EL ESPACIO PÚBLICO}

Entre los arquitectos españoles contemporáneos con proyección internacional, es probablemente José Ignacio Linazasoro uno de los que ha dedicado mayor atención al espacio público urbano. En este sentido, el proyecto para la reordenación de las inmediaciones de la catedral de Reims (2003-2008) ocupa un lugar del todo especial dentro de la obra del arquitecto donostiarra; de él ha dicho que "está entre los más difíciles e interesantes con que me he encontrado"4. Por otra parte, el lugar en que se ubica está entre los más emblemáticos de Francia, habida cuenta de que la catedral de Reims fue durante siglos el escenario de una ceremonia de gran significado histórico para la monarquía gala, pues allí se celebraba la consagración de sus reyes (figura 1).
El encargo de la plaza de Reims le llegó a Linazasoro como consecuencia de un doble concurso, del que resultó ganador. El hecho de que entre ambos concursos mediaran diez años explica quizá que sea uno de sus proyectos más meditados y con un mayor tiempo de maduración, a lo que se ha de añadir la experiencia que ha ido acumulando su autor en actuaciones de escala y naturaleza urbana, todas ellas planteadas a partir de una particular atención a la preexistencia y al contexto. No en vano, la suya fue definida por Marchán Fiz como "una arquitectura con voluntad de adecuación" ${ }^{5}$, y sus proyectos centrados en la ordenación de espacios públicos no han hecho sino confirmarlo. Me referiré aquí únicamente a los que considero relacionados de algún modo con la actuación de Reims, omitiendo otros, también importantes, pero de menor interés para el caso.

De todos modos, conviene no perder de vista que esa atención al espacio público ha sido una constante en la trayectoria de Linazasoro desde sus inicios; pensemos, por ejemplo, entre los proyectos de los años 70, realizados con Miguel Garay, en la reordenación de la antigua plaza de San Juan en Irún (1975), en la primera versión del cementerio de Vergara (1976) o en la propuesta para el concurso de una plaza en Legazpi (1979). En todos ellos, con un lenguaje neorracionalista fruto del deslumbramiento que la Tendenza produjo en muchos de los jóvenes arquitectos de aquel momento, aparecen ya algunas cuestiones que van a resultar centrales en sus proyectos posteriores: el interés por el vacío urbano, el énfasis en el diseño de los pavimentos y la atención a las arquitecturas del entorno como estrategias para dar un significado al lugar.

Pero son sobre todo dos proyectos de muy distinta naturaleza, redactados ambos en 1982 por Linazasoro ya como autor único, los que hicieron de laboratorio donde experimentar con algunas de las ideas fundamentales que luego aplicaría en Reims; me refiero a la reforma del ámbito de las antepuertas del santuario de Loyola y

3. En el contexto español, cabría recordar las operaciones de aislamiento de las catedrales de León y Burgos, llevadas a cabo a comienzos del siglo XX, aunque hubieran sido concebidas algunas décadas antes, siguiendo lo ya realizado en tantas catedrales francesas.

4. LINAZASORO, José Ignacio. Memoria de una búsqueda. Sobre escritos y proyectos. Valladolid: Universidad de Valladolid, 2019, p. 71.

5. MARCHÁN FIZ, Simón. Una arquitectura con voluntad de adecuación. En: J. I. Linazasoro. Barcelona: Gustavo Gili, 1989, pp. 6-19. 
1. Pierre-Denis Martin el Joven. Cabalgada de Luis XV tras la consagración el 26 de octubre de 1722 (1724). Museo de Versalles.

2. José Ignacio Linazasoro. Antepuertas del santuario de Loyola (1982-1985).

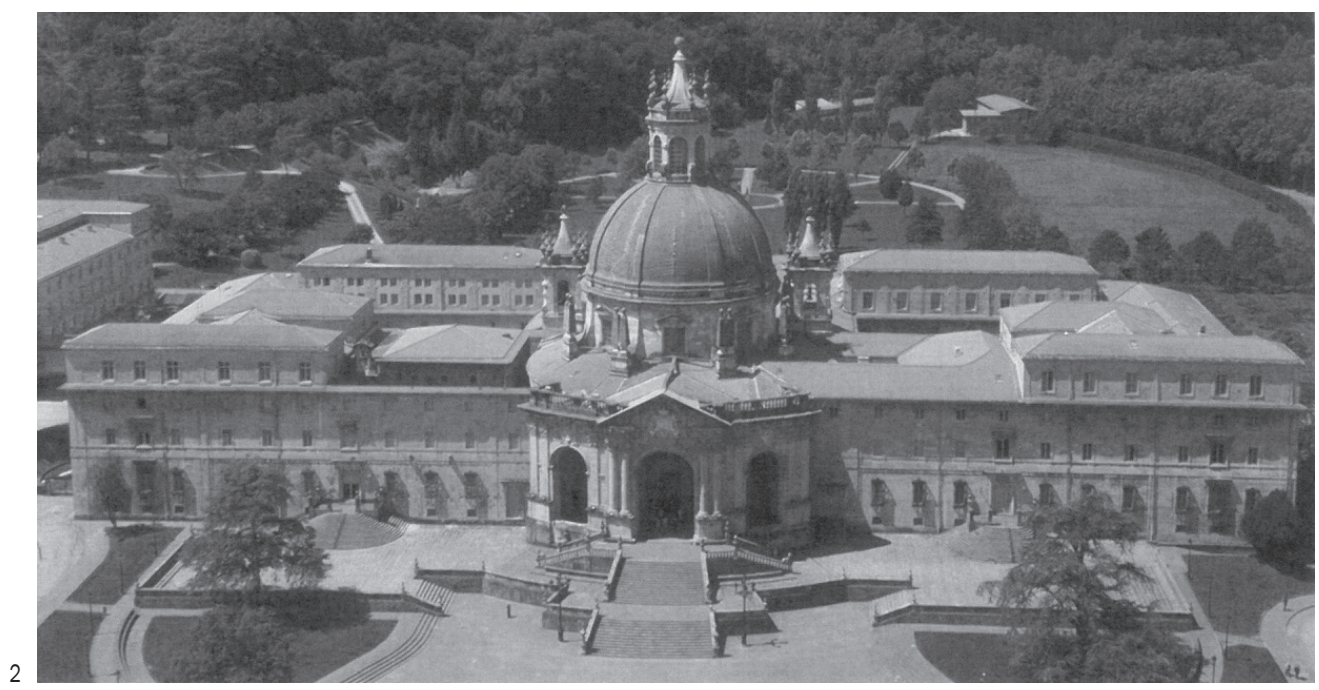

a la remodelación de la plaza de Balda, en Azcoitia. De ellos dijo que habría que verlos como "la afirmación de otra modernidad no identificable con la derivada de las vanguardias, desde la que se propone la reutilización de todo un repertorio de formas e instrumentos proyectuales heredados de la tradición [...] que lejos de resultar obsoletos resultan, finalmente, los más adecuados en la recuperación de una imagen arquitectónica [...] alejada de todo experimentalismo"6. Eran los años de la publicación de El proyecto clásico de la arquitectura (1981) y de la recuperación por parte del arquitecto donostiarra de unas formas enraizadas en la historia.

De ahí viene probablemente ese interés suyo por trabajar con las preexistencias, por considerar que los problemas a los que nos enfrentamos ya han sido afrontados por otros y por buscar en sus soluciones una referencia para el proyecto ${ }^{7}$. En el caso de la actuación en el entorno de Loyola, nos encontramos ante una propuesta que intenta no solo adecuar el santuario diseñado por Carlo Fontana a un entorno que en gran medida le resultaba ajeno, sino, sobre todo, encajarlo en el lugar conforme a premisas ya contenidas en el proyecto original. Desde esta perspectiva, y habida cuenta del carácter "urbano" de la arquitectura del santuario, se trataba, en primer lugar, de resolver su relación con el suelo. La plataforma proyectada inicialmente por Linazasoro como soporte del conjunto monumental quedó luego reducida a dos lonjas simétricas de menor tamaño, que tenían la función de resolver el encuentro de la escalera monumental con el espacio situado inmediatamente delante del edificio (figura 2).

El recurso a un pavimento de piedra, cuyo despiece es minuciosamente diseñado, adelantaba ya una constante en los sucesivos proyectos de Linazasoro relacionados con el espacio público, en los que la materialidad y la vegetación son los elementos básicos para asegurar la atemporalidad y el anonimato de la solución finalmente elegida. Es esto lo importante de este proyecto, más allá de que el lenguaje elegido sea todavía

6. LINAZASORO, José Ignacio. Cuatro proyectos. En: Arquitectura, 1983, n. ${ }^{\circ} 245$, p. 53.

7. Esa "negociación con lo ya construido" se lleva a cabo en relación "con la arquitectura en su historia como caudal y acervo, como el ámbito en el que la producción de nueva arquitectura encuentra una privilegiada situación de continuidad y de estímulo" (SOLÀ-MORALES, Ignasi de. Imitación esencial. La arquitectura de José Ignacio Linazasoro. En: Efrén GARCÍA GRINDA, ed. J. I. Linazasoro. Obras y proyectos, 1988-97. Madrid: Fundación Argentaria, 1998, p. 10). 


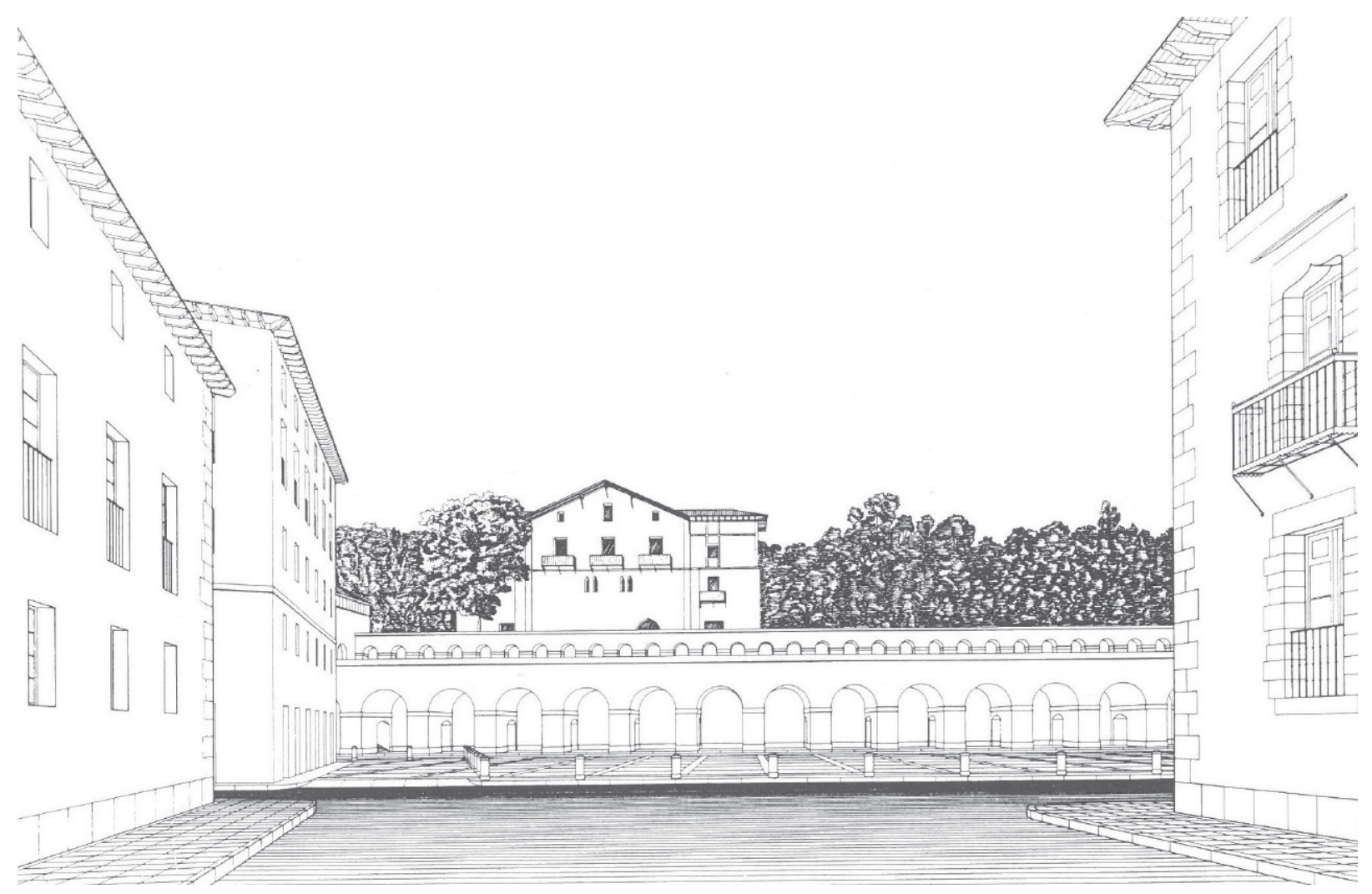

3

vagamente clasicista, algo que también sucede con la primera versión de la plaza de Balda, en Azcoitia, donde la idea de orden contenida tanto en la repetición de la arquería como en la regularidad de la cuadrícula del pavimento remitía igualmente a una figuratividad centrada aún en cuestiones compositivas ligadas al clasicismo (figura 3); más concretamente, a la plataforma que sirve de soporte para la implantación de El Escorial, una cuestión de la que el arquitecto donostiarra ya se había ocupado "desde un punto de vista teórico" en un artículo anterior ${ }^{8}$.
Además, en ambos proyectos estaba ya presente otra cuestión, que es clave para el argumento que aquí se quiere desarrollar y sobre la que Linazasoro volverá más adelante; me refiero al entendimiento del espacio público como una "arquitectura expandida", en el sentido de que su proyecto integra dentro de una unidad superior determinadas arquitecturas inmediatamente relacionadas con él. En Loyola, la explanada y el jardín que el arquitecto donostiarra diseñara ante el santuario -aunque no ejecutados- pretendían "continuar la idea que derivaría del propio proyecto del edificio", tal como había sido planteado

8. LINAZASORO, José Ignacio, op. cit., supra, nota 6, p. 54; el artículo mencionado es LINAZASORO, José lgnacio. El Escorial y la arquitectura del clasicismo. En: Carrer de la Ciutat, 1978, n. ${ }^{\circ}$ 5, pp. 5-10.

9. LINAZASORO, José Ignacio. Proyecto de ordenación del área del santuario de Loyola. En: Revista Internacional de Estudios Vascos, 1983, vol. 28 , n. ${ }^{\circ} 2$, p. 472. 
por Fontana. En Azcoitia se trataba, en cambio, de permitir un diálogo más fluido y articulado entre las casonas de Balda e Irízar a través de una arquitectura que, a la vez que construía la plaza, resolvía los problemas derivados de la compleja topografía del entorno.

Una estrategia proyectual semejante la encontramos en una actuación histórica estudiada por Linazasoro en esos años: la Casita del Príncipe, diseñada por Juan de Villanueva en las inmediaciones de El Escorial ${ }^{10}$. Se trata de un pequeño pabellón de recreo, compuesto por un primer cuerpo construido entre 1771 y 1775, luego ampliado hacia la zona de poniente con un "salón, ensanche del jardín y estanque" entre 1781 y 1785. La estrecha relación entre este nuevo espacio interior y el jardín trasero, ejecutados simultáneamente, venía exigida por el uso recreativo del edificio; de hecho, probablemente fuera la insuficiencia de las pequeñas salas disponibles en el cuerpo delantero lo que motivó la ampliación, con el fin de disponer de un lugar apto para el esparcimiento, intermedio entre el pabellón y el bosque de robles circundante. El conjunto "mantiene un orden superior inalterado, un procedimiento compositivo que, finalmente, se enriquece y complejiza al hacerse más expresivo por manifestar sus contenidos con mayor claridad al exterior"11.

\section{REIMS, LUGAR DE MEMORIA}

A la vez que hacía esos proyectos para Loyola y Azcoitia, Linazasoro se ocupó, como parte de su tarea docente, del entorno de la catedral de Notre-Dame de París, planteando a los estudiantes un ejercicio centrado en la reordenación del vacío urbano que la rodeaba. Este trabajo le llevó a profundizar en la doble actuación llevada a cabo en el siglo XIX: de una parte, la "restauración" de la catedral planteada por Viollet-le-Duc siguiendo la racionalidad de unos principios estilísticos que debían repristinar su imagen y, de otra, la destrucción del tejido medieval circundante con el fin de configurarla como un monumento aislado; todo ello, para escenificar ese gran relato nacional que vio en las catedrales góticas un símbolo de la unidad del pueblo francés. Con ello, el arquitecto donostiarra pudo también familiarizarse con los debates, desarrollados en Francia durante la segunda mitad del siglo $X X$, en torno a los posibles modos de reconfigurar las inmediaciones de los espacios que rodeaban a esas grandes catedrales ${ }^{12}$.

Esos conocimientos le iban a servir para enfrentarse al primero de sus proyectos franceses, que le llegó a través de un concurso de ideas para reordenar el entorno de la catedral de Reims, convocado en 1991 y del que resultaría ganador ${ }^{13}$. Esta ciudad no solo constituía un relevante lieu de mémoire para la nación francesa, sino que, además, el objeto mismo del concurso se hallaba referido al principal de sus monumentos, la catedral. Y es que tanto una como otra estaban asociadas, en la memoria de los franceses, a una ceremonia del todo particular: la consagración de sus monarcas, celebrada allí desde los tiempos de la dinastía carolingia ${ }^{14}$. No obstante, con el paso del tiempo el entorno de la catedral había experimentado importantes cambios, configurando un espacio urbano en el que prevalecieron la axialidad y el dégagement, rompiendo la relación orgánica que históricamente había existido entre la catedral y su entorno.

10. LINAZASORO, José Ignacio, El arte de la imitación en Juan de Villanueva. La Casita del Príncipe en El Escorial. En: Arquitectura, 1982, n. ${ }^{\circ} 239$, pp. 68-73. El artículo está centrado en cuestiones relacionadas con la tradición clásica en torno a la cual giraba entonces la investigación linazasoriana, pero no resulta difícil intuir que al arquitecto donostiarra debió interesarle de manera particular el refinado planteamiento de conjunto, que integra interior y exterior de un modo tan brillante.

11. MOLEÓN, Pedro. El arquitecto Juan de Villanueva (1739-1811). Madrid: Akal, 2020, p. 13.

12. Para comprobarlo, basta con repasar las actuaciones recogidas en JAKOVLEVIC, Nada; CULOT, Maurice, dirs. Places et monuments. Bruselas: Mardaga, 1984, entre las que figuran la propuesta de Linazasoro para la plaza de Azcoitia (pp. 78-81) y dos de los proyectos del curso desarrollado con los estudiantes de la Escuela de San Sebastián (pp. 158-163).

13. Además de la plaza de la catedral de Reims, Linazasoro ha construido en Francia un centro de congresos en Troyes (2010-14) y, más recientemente, ha ganado los concursos para la ordenación del entorno de las basílicas de Notre-Dame-du-Port, en Clermont-Ferrand (2018) y Notre-Dame-des-Miracles, en Mauriac (2019). 14. Puede verse al respecto LE GOFF, Jacques. Reims, ville du sacre. En: Pierre NORA, ed. Les lieux de mémoire, vol. II-1: La Nation. París: Gallimard, 1986, pp. 89-194. 


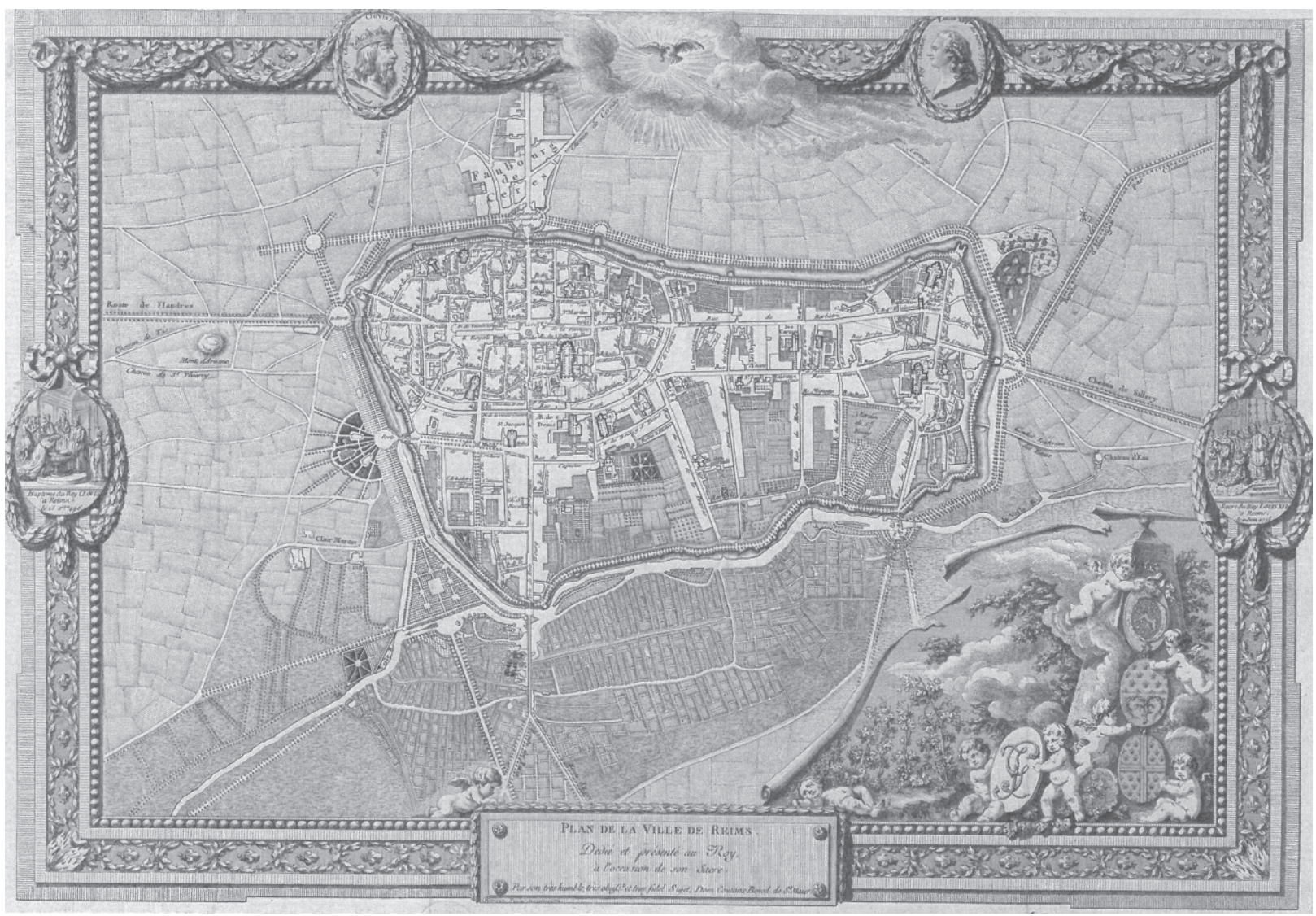

Los primeros cambios en el tejido medieval de Reims se produjeron como consecuencia del plan de embellissement diseñado en 1754 por el ingeniero Jean-Gabriel Legendre (figura 4), donde ya se planteaba la posibilidad de abrir una plaza delante de la catedral. En el siglo XIX tuvo lugar la apertura de la calle Libergier, como eje de perspectiva que unía la catedral con el canal (figura 5), así como la sustitución del histórico Hôtel-Dieu, un antiguo hospital, por el actual Palacio de Justicia, ampliando con ello el espacio del parvis. Pero la gran transformación de la zona es debida a los bombardeos de la Primera Guerra Mundial, de resultas de los cuales quedaron destruidas muchas de las edificaciones que rodeaban la catedral ${ }^{15}$. El plan de reconstrucción ideado en los años 20, en continuidad con los planteamientos decimonónicos, sirvió para dejar exenta su cabecera.
Ya en los años 60, en el contexto de la reflexión desarrollada en Francia sobre el entorno de los monumentos de carácter patrimonial ${ }^{16}$, se pensó en intervenir en los alrededores de la catedral, aunque los criterios para hacerlo no se fijarían hasta bien entrados los años 80 , en el marco de la renovación del centro histórico de Reims. Los debates habidos en todo el país estuvieron centrados en la oportunidad, o no, de reocupar con edificaciones los vacíos que rodeaban las catedrales y sobre la arquitectura más conveniente para hacerlo. En Reims hubo fuertes protestas ciudadanas a mediados de los años 70 ante la posibilidad de construir frente a la catedral un edificio de hormigón y cristal, destinado a ser la sede del Tribunal de Apelación, que finalmente se situó en un lugar menos comprometido desde el punto de vista patrimonial. No fue un caso aislado; algo semejante sucedió en Rouen y Amiens.

15. Véase BENDITO, María. La basílica devastada: el bombardeo de la catedral de Reims y la identidad nacional del arte gótico. En: Acta Artis. Estudis d'Art Modern, 2017, n. ${ }^{\circ} 4-5$, pp. 241-263.

16. Sobre la actuación de la Commission des Abords en esos años, véase BACKOUCHE, Isabelle. Expertiser la rénovation urbaine. Le cas de la France dans les années 1960. En: Genèses, 2008, n. ${ }^{\circ}$ 70, pp. 45-65. Para el contexto de los debates patrimoniales puede ser útil la consulta de LAURENT, Xavier. Grandeur et misère du patrimoine d'André Malraux à Jacques Duhamel (1959-1973). París: École des Chartes, 2003, en especial pp. 147-162, dedicadas a la cuestión de los abords. 
4. Jean-Gabriel Legendre. Plan general de la ciudad de Reims (1754).

5. La calle Libergier antes de los derribos para la rectificación de sus alineaciones (1894).

Fotografía de los hermanos Varin.
El conflicto de Amiens puede resultar emblemático de los debates habidos en esos años. La polémica comenzó con la construcción a comienzos de los años 70 de la llamada maison de verre, un edificio pretendidamente miesiano emplazado en las inmediaciones de la catedral ${ }^{17}$. En la década siguiente se convocó un concurso restringido para reordenar la zona de la catedral, que fue ganado por Rob Krier (figura 6). Con un enfoque netamente morfologista, el arquitecto luxemburgués proponía recuperar la escala de la ciudad medieval a través de la construcción de un conjunto de arquitecturas, que configuraban una serie de espacios públicos concatenados, reconfigurando así la estructura del antiguo parvis ${ }^{18}$. La propuesta no llegaría a ejecutarse, haciéndose cargo del planeamiento de ese entorno urbano el arquitecto francés Bernard Huet, quien levantaría a finales de los años 90 un edificio frente a la catedral que ocultaba la vista del edificio de cristal preexistente ${ }^{19}$.

Linazasoro, que estaba familiarizado con estos planteamientos, elegiría para su proyecto de Reims un enfoque del todo diferente, en el que el protagonismo correspondería a la cualificación del espacio público. Ni la propuesta de Krier ni la de Huet le convencían; a su entender, no eran otras arquitecturas lo que se necesitaba para resolver el problema del vacío surgido en torno a la catedral: de hecho, lo que él proponía en Reims era redefinir la forma de la plaza sin modificar su tamaño. Consideraba que las propuestas de Amiens iban "en contra de un proceso que, aunque había sido en cierto modo negativo, resultaba ya irreversible, pues no era lógico reducir las dimensiones de un espacio público que había sido ganado con el derribo de las antiguas construcciones"20. Se hallaba, pues, más próximo al enfoque paisajista del proyecto de Oriol Bohigas para Amiens, que pivotaba sobre el tratamiento del vacío como parte de una red de

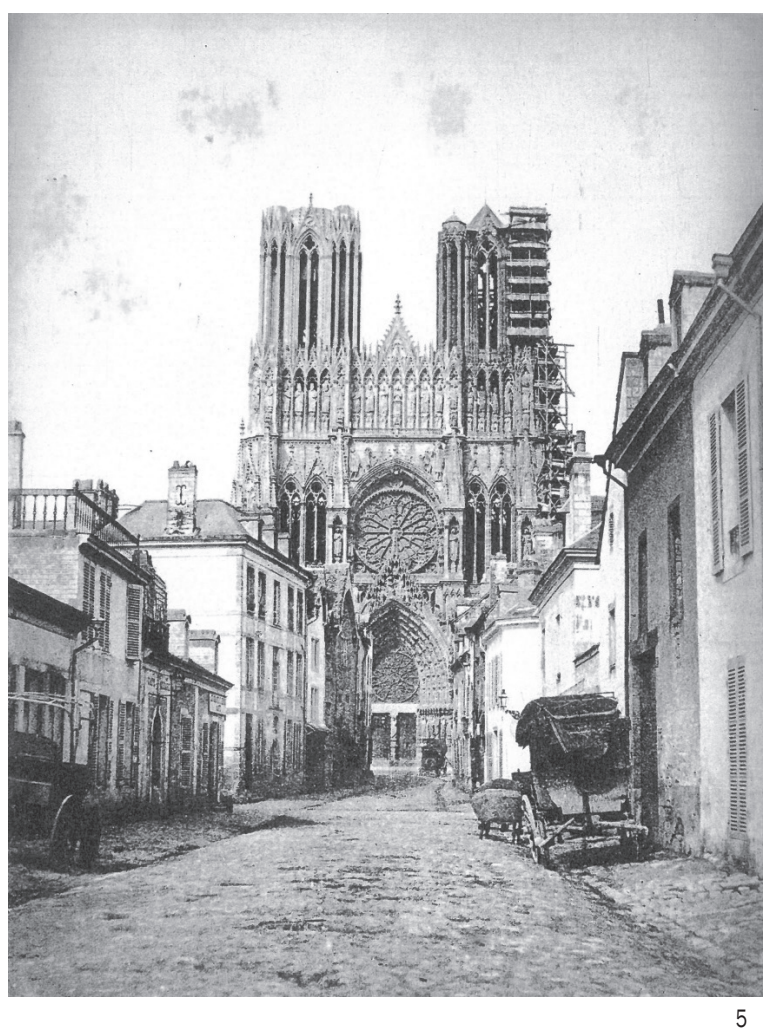

espacios libres que definir por la actuación ${ }^{21}$. El hecho de que el vencedor de aquel concurso fuera Krier indica cuáles era las ideas dominantes al respecto en el contexto francés de esos años.

La intención de Linazasoro en Reims era clara: "Se trataba de recuperar la escala perdida y, sin pretender acometer la tarea imposible de reconstruir el tejido urbano desaparecido, proyectar un entorno que mediante un nuevo trazado y una geometría más adecuada permitiera recuperar el antiguo equilibrio entre monumento y ciudad"22. Con ello, el parvis volvía a entenderse como una parte integrante del monumento, que formaba parte de la memoria del lugar y resituaba la catedral en un entorno capaz de posibilitar una percepción de la misma más acorde con la experiencia de la ciudad medieval, donde había surgido. Tal vez sea este el motivo por el que el arquitecto donostiarra haya señalado que su propuesta estaba pensada con el fin de que "pudiera servir de paradigma

17. El punto de vista del arquitecto del proyecto puede verse en BOUGEAULT, Bernard. La maison de verre d'Amiens. Son histoire vraie. París: Books on Demand, 2018. 18. Véase KRIER, Rob. Aménagement du Secteur Nord du centre de la ville d'Amiens. En: UR, 1985, n. ํㅡ 2, pp. 2-7.

19. Un resumen de los debates habidos con ese motivo en ROSEMBERG, Muriel. Questions sur un conflit d'aménagement: le parvis de la cathédrale d'Amiens. En: Patrice MELÉ; Corinne LARRUE; Muriel ROSEMBERG, dirs. Conflits et territoires. Tours: Presses Universitaires François-Rabelais, 2004, pp. 165-186.

20. LINAZASORO, José Ignacio, op. cit., supra, nota 4, p. 54.

21. Véase MARTORELL, Josep; BOHIGAS, Oriol; MACKAY, David. La nouvelle Amiens. En: UR, 1985, n. ${ }^{\circ}$ 2, pp. 10-15.

22. LINAZASORO, José Ignacio. Textos críticos. Madrid: Asimétricas, 2017, pp. 111-112. 


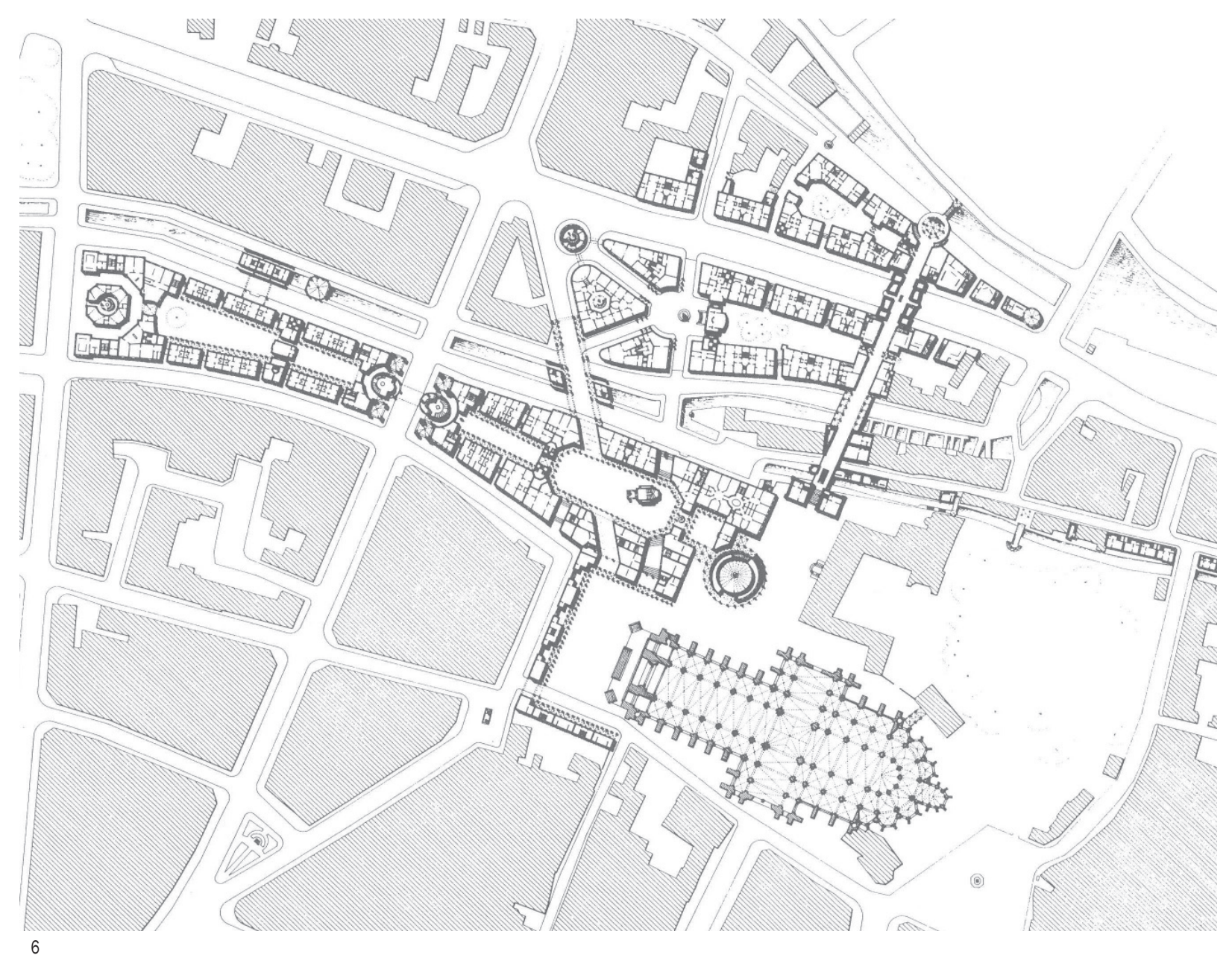

para otro tipo de intervenciones semejantes en la propia Francia"23.

\section{UN PROYECTO CONTEMPORÁNEO PARA LA PLAZA DE LA CATEDRAL}

En la primera mitad de los años 90 Linazasoro viajó a los países nórdicos y pudo visitar determinadas obras de arquitectos como Aalto, Asplund y Lewerentz, que siempre le habían atraído. El conocimiento de primera mano de la Universidad de Jyväskyla, de la casa de Muraatsalö o del Cementerio del Sur de Estocolmo, por citar algunas que son relevantes para nuestro argumento, lo llevó a profundizar en una serie de cuestiones de las que ya se había ocupado, pero que comenzaron a estar presentes de un modo nuevo en sus proyectos; me refiero, entre otras, a la importancia de la materialidad, a la interacción interiorexterior de los edificios o a su modo de implantarse en el lugar. Y es que, de la arquitectura de ese conjunto de maestros, le interesó sobre todo "su carácter urbano y fragmentario, además de matérico, y en ese sentido, mucho más que sus formas, [le interesaron] sus referencias, que se relacionaban con algunos intereses permanentes de [su] trabajo como las ciudades históricas y el proyecto urbano"24.

En este contexto, la versión finalmente construida del proyecto de Azcoitia (1997-1999) experimentaría una sustancial simplificación, que expresa bien este renovado enfoque de la arquitectura de Linazasoro. El protagonismo de las preexistencias queda ahora subrayado por la anónima discreción de los elementos que el proyecto introduce: el pavimento, menos rígido y más contextual; los muros, netamente definidos por su materialidad; el basamento que sirve de soporte a una escultura de Oteiza y que es, a la vez, un banco donde sentarse (figura 7). 
6. Rob Krier. Planta de la propuesta para el entorno de la catedral de Amiens (1984).

7. José Ignacio Linazasoro. Plaza de Balda, Azcoitia (1997-99). Fotografía de Javier Azurmendi.

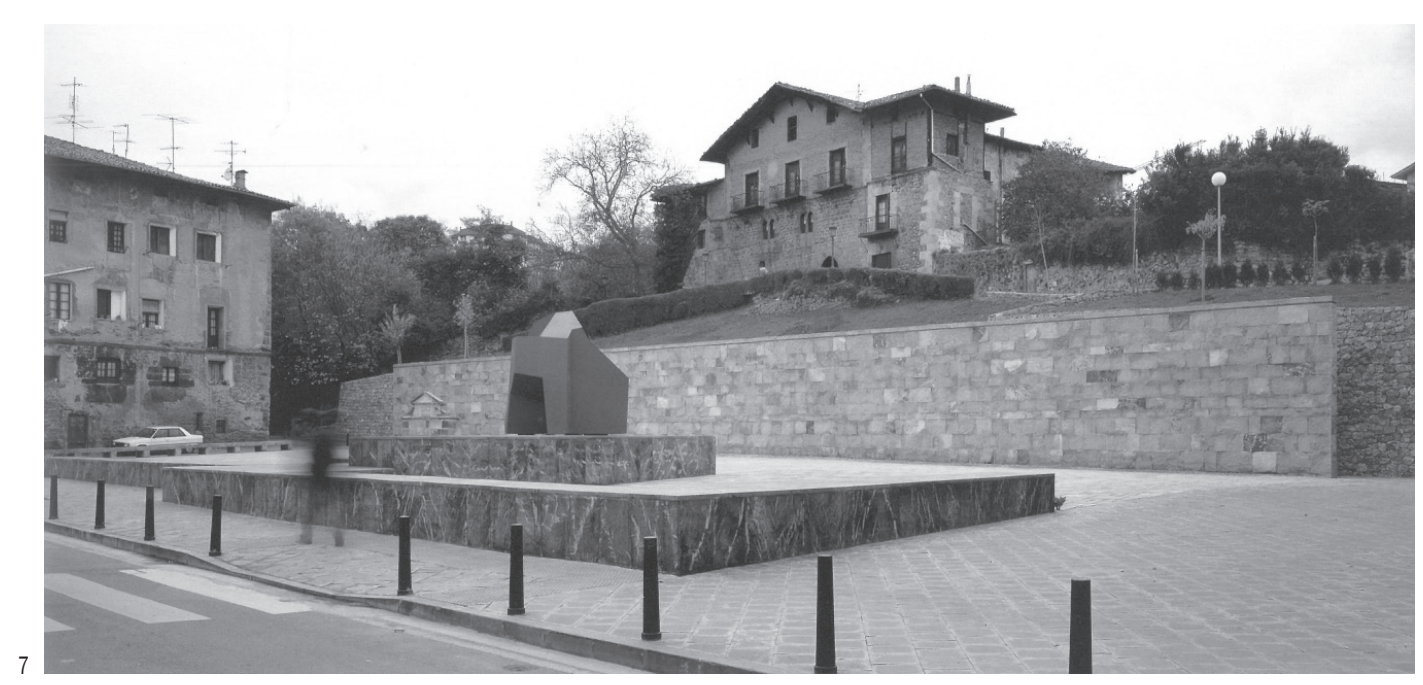

Se genera así un espacio netamente contemporáneo, en el que el acento está puesto en la abstracta geometría empleada en su trazado y en los materiales utilizados en su construcción: "Tenía en ese momento presente - ha dicho su autor - las dos plazas donostiarras de Luis Peña, en particular la de la Trinidad, que consideraba modélica de la búsqueda de anonimato y atemporalidad que caracterizan los espacios públicos"25.

Ciertamente, la plaza de la Trinidad (1963) constituye un claro ejemplo de cómo completar y articular, mediante el espacio público, un conjunto informe de arquitecturas de muy diferente condición -incluido el frontón preexistente-, situadas en un contexto complicado en lo topográfico y degradado en lo físico. Mediante el recurso a la materialidad, Peña consiguió "alcanzar unidad y armonía con un coro de voces bien diversas [...], mostrando que hacer arquitectura es también establecer un diálogo con lo que se construyó en el pasado, convertir en realidad tangible y dotar de nuevo significado a elementos que lo habían perdido"26.
En la misma línea cabe situar las cuatro plazas de Guimarães (1987-1995), proyectadas por Fernando Távora en el centro histórico de esa ciudad portuguesa. También en este caso se recurre a los empedrados y a pequeños elementos de mobiliario urbano, cuyo diferente modo de componerse y organizar el espacio público responde al diverso carácter de la arquitectura de cada plaza, que de algún modo prolongan e interpretan: "Así, es barroco el carácter de la plaza del Ayuntamiento, medieval el de la plaza de Santiago, renacentista el del largo de João Franco y romántico el del largo de la Condesa de Juncal. Diferentes caracteres insertados en un recorrido urbano que garantiza su unidad dentro de su diversidad"27.

La expresión decididamente actual del lenguaje adoptado por ambos arquitectos y el énfasis puesto en el tratamiento del suelo no están lejos de la solución adoptada por Linazasoro en Azcoitia, donde los pavimentos se configuran como "metáfora llena de significados, que se asume tanto por la espacialidad que instituye como por la urbanidad que intuye"28. En todos estos casos, pero

25. LINAZASORO, José Ignacio, op. cit., supra, nota 4, p. 55.

26. MONEO, Rafael. Cuando la arquitectura quiere ser paisaje. En: Rocío PEÑA; Mario SANGALLI, eds. Luis Peña Ganchegui. Arquitecturas 1959-1994. San Sebastián: Fundación Kutxa-Universidad del País Vasco, 1994, p. 12.

27. TÁVORA, Fernando. Rehabilitações urbanas. Guimarães. En: Luiz TRIGUEIROS, ed. Fernando Távora. Lisboa: Blau, 1993, p. 178.

28. NASCIMENTO OLIVEIRA, Francisco. Chão da cidade: permanencia e transformação. De metáfora a impressão digital da cidade. En: Proyecto, Progreso, Arquitectura, 2011, n. ${ }^{\circ}$, p. 140. 


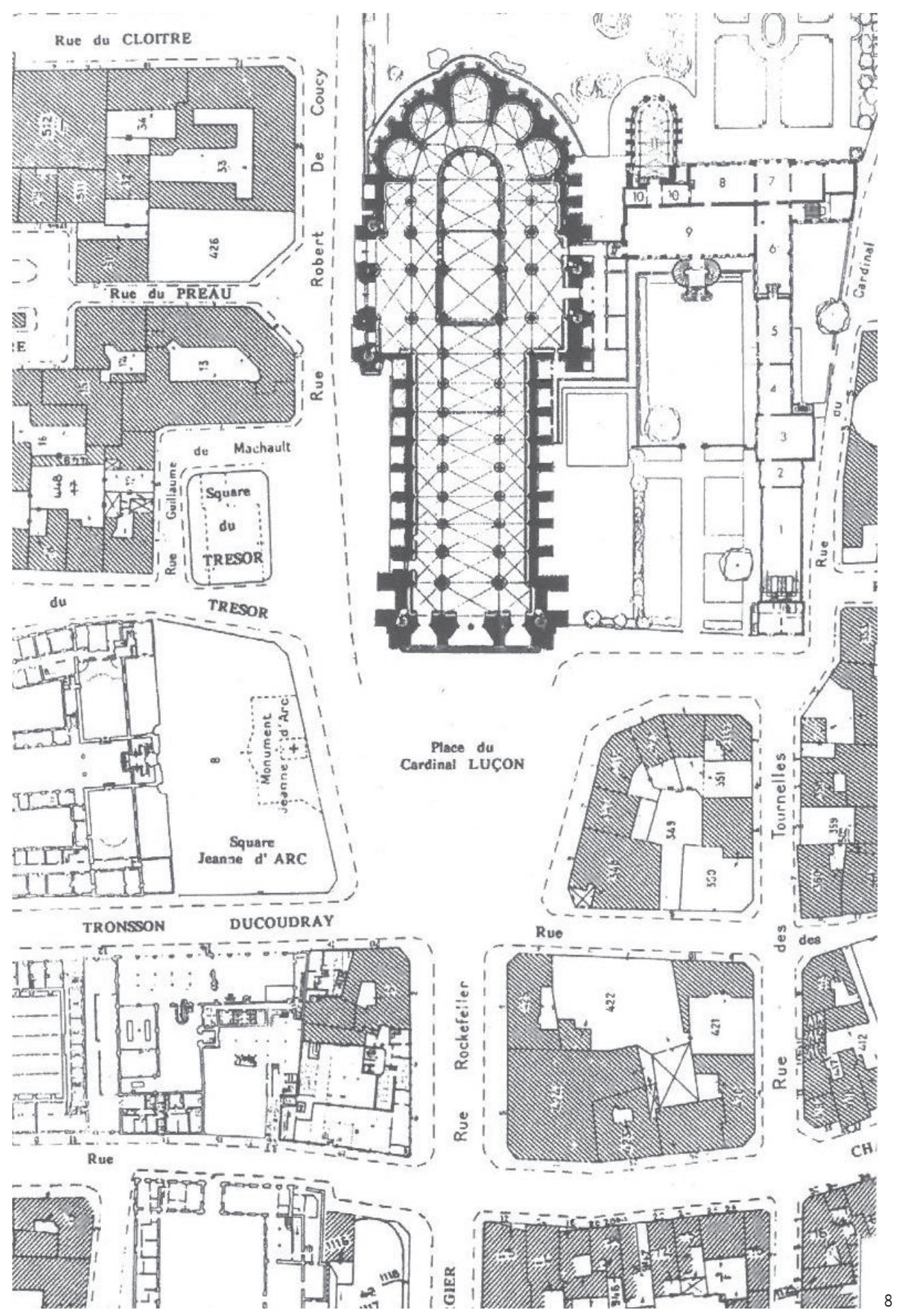


también en la plaza de Reims, el suelo viene a ser el tapiz donde se tejen los hilos antiguos de unas arquitecturas preexistentes con otros hilos completamente nuevos, legitimando así las decisiones creativas tomadas en el diseño de los pavimentos, cuya materialidad aparece como una expresión del paso del tiempo. Y es que, como ha afirmado a este respecto Navarro Baldeweg, "las metas de cualquier proyecto no se satisfacen, o no solo, en la belleza de un objeto, sino en la capacidad funcional de dar cita a sensaciones y emociones esenciales de una vida anterior"29.

En el caso de Reims, esa vida "anterior" fue buscada por Linazasoro en la historia de la ciudad; el enfoque urbano de su propuesta venía exigido por el modo en que estaba planteado el primer concurso de ideas para el entorno de la catedral, convocado en 1992. El arquitecto donostiarra propuso una reconsideración de conjunto que diera un nuevo sentido al vacío que antecedía a la gran fachada gótica, retornando a los valores de la herencia medieval perdida con una actitud netamente contemporánea. Su propósito era sacar a la catedral del aislamiento en que se hallaba (figura 8). Para ello, decidió cambiar la orientación del espacio del parvis y abrirlo a nuevos ejes urbanos que hicieran posible la aparición de vistas oblicuas de la catedral. El diseño de la plaza se lleva a cabo, tras las huellas de Camillo Sitte, a partir de las lecciones aprendidas en las plazas de las ciudades históricas italianas, aquí reinterpretadas.

Los análisis de Sitte ya ocupaban un lugar importante entre las referencias de Places et monuments ${ }^{30}$; en particular, es fácil identificar las imágenes de la plaza del Campo de Siena o de la Señoría de Florencia, ahí recogidas, como la fuente de determinadas decisiones proyectuales de Linazasoro, entre las que cabría señalar la importancia otorgada a la topografía y al dibujo del pavimento o el desplazamiento de la estatua de Juana de Arco para colocarla en una posición que le permita articular las diferentes secuencias espaciales que la propuesta contiene. Pero es, sobre todo, en el discurso en torno al vacío donde la influencia del arquitecto vienés se revela determinante: "Sitte demuestra -ha dicho Linazasoro a este respecto- que en las ciudades antiguas el espacio es esencial, hecho con pocas cosas bien situadas y completamente flexible desde el punto de vista funcional. Cuando proyecté la plaza de Reims, tenía en mente precisamente esto"31.

El concurso de 1992 buscaba establecer un marco de referencia para las posteriores intervenciones concretas en el entorno de la catedral. En ese contexto, en 1997 se convocó otro concurso, esta vez para definir la mediateca que había de construirse en la embocadura de la calle Rockefeller, sobre el solar que ocupara el antiguo edificio de la Policía. Linazasoro presentó, junto con el estudio Thiénot-Ballan, un proyecto que no resultaría vencedor, pero que le permitió seguir reflexionando sobre el lugar. No sería hasta 2003 cuando se convocara el concurso para definir la plaza situada frente a la catedral, que volvió a ser ganado por Linazasoro, también asociado esta vez con el mencionado estudio remense. Es entonces cuando se reformula y actualiza el primer proyecto, produciendo "una versión más esencial, con una geometría más clara aunque siempre irregular para no competir con la geometría de la catedral"32 (figuras 9 y 10).

La idea era devolver la catedral a un entorno cuya escala se adaptase a la del parcelario medieval desaparecido, rescatado ahora mediante una estrategia proyectual que combinaba los taludes y la vegetación para rememorar, de manera análoga, su anterior estructura. Se creaba para ello una sutil topografía artificial que intentaba dar al espacio público la forma del entorno urbano precedente, acudiendo a una geometría similar a la del trazado medieval (figura 11). Se conformaron así cuatro espacios diferenciados: "Uno central que se corresponde con la

29. NAVARRO BALDEWEG, Juan. El tapiz, unas visiones y un proyecto. En: REIA. Revista Europea de Investigación en Arquitectura, 2021, n. 17, p. 13. El término "tapiz" equivale a "contexto"; la defensa que aquí se hace de este sintoniza bien, aunque los acentos sean ciertamente distintos, con la llevada a cabo por Linazasoro en su obra.

30. En JAKOVLEVIC, Nada; CULOT, Maurice, dirs., op. cit., supra, nota 12, pp. 47-55, se recogía el primer capítulo del célebre tratado del urbanista austríaco, con ilustraciones procedentes de la edición francesa de 1918.

31. GUARRERA, Fabio, ed. Trentasette domande a José lgnacio Linazasoro. Nápoles: CLEAN, 2014, p. 39.

32. LINAZASORO, José lgnacio, op. cit., supra, nota 9, p. 69. 

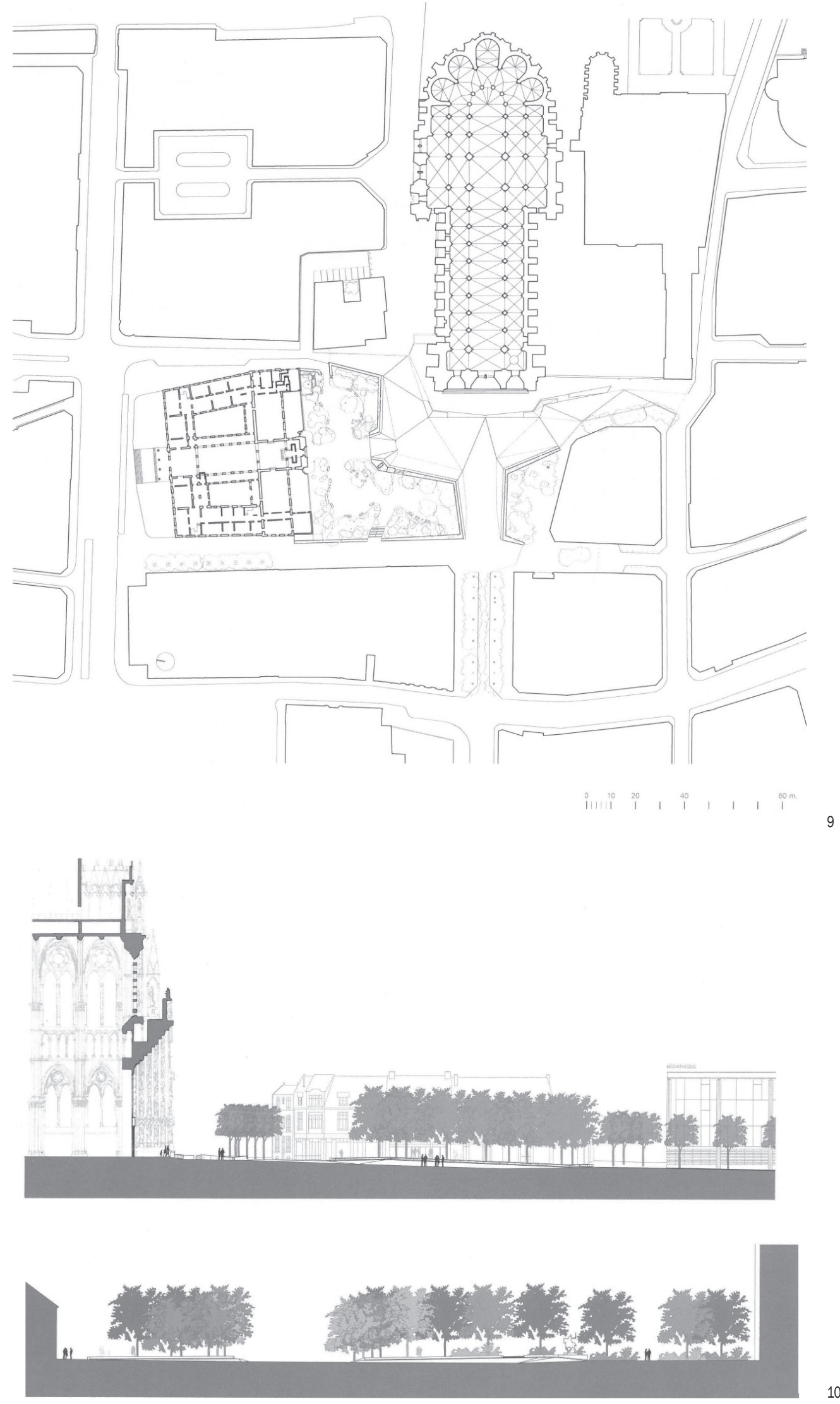
9. José Ignacio Linazasoro. Planta con la ordenación definitiva de la plaza de la catedral de Reims (2005). 10. José Ignacio Linazasoro. Secciones longitudinal y transversal de la plaza de la catedral de Reims (2005).

11. El espacio central de la nueva plaza visto desde la catedral de Reims. Fotografía de Nicolas Waltefaulge.

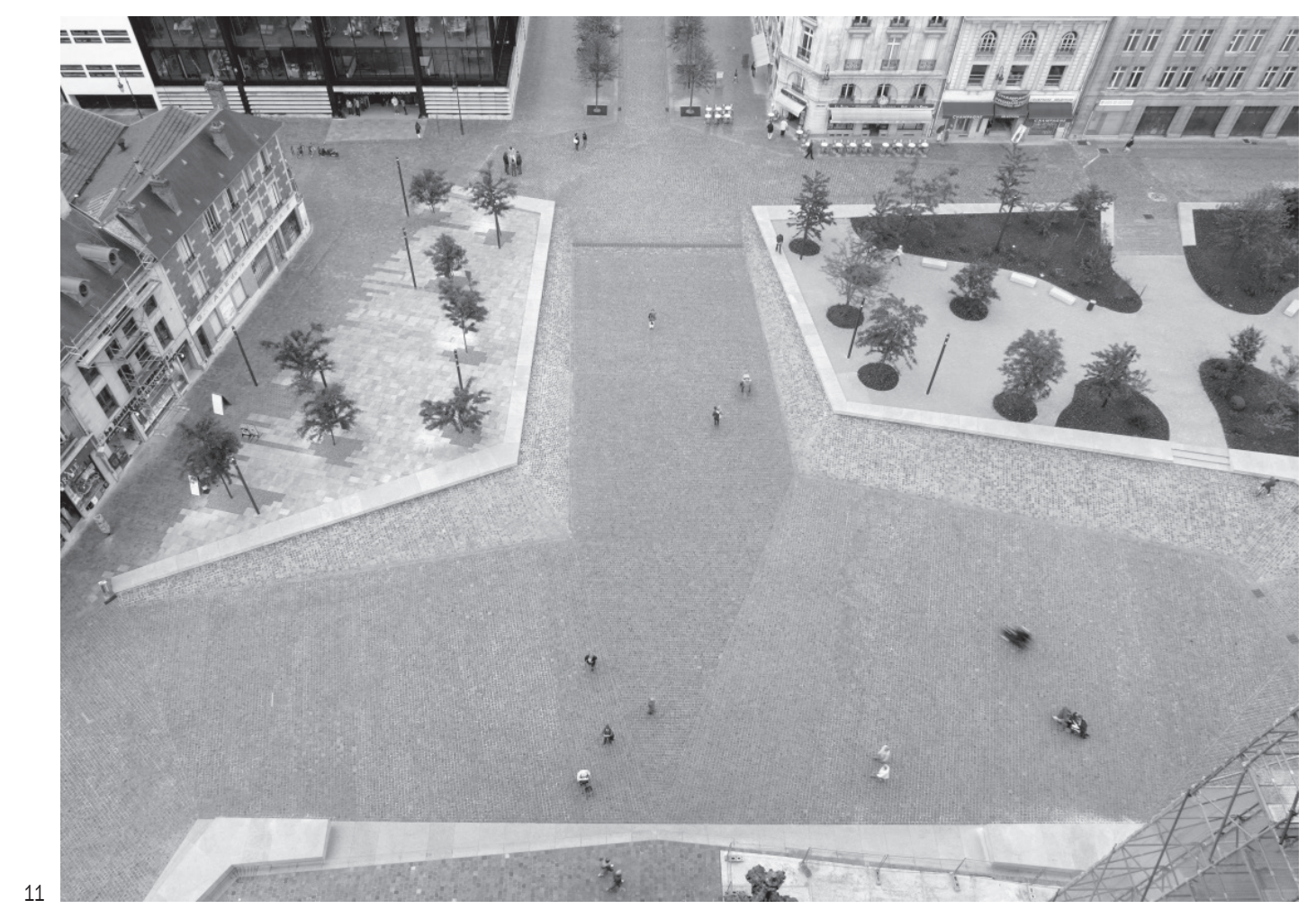

plaza propiamente dicha, dos laterales en forma de plataforma que evocan, más o menos literalmente, la forma de las manzanas desaparecidas, cada una con distinto tratamiento, y un parvis o sagrato junto a la fachada que recuperaba un espacio perdido en el siglo XVIII"33. Toda la operación aspira a recrear un pasado apócrifo como si de una permanencia arqueológica se tratara.

El proyecto se simplificaría durante la fase de ejecución, unificando los recursos expresivos destinados a crear los diferentes ámbitos espaciales en que ha quedado fragmentado el inhóspito vacío preexistente. En lo que a la materialidad se refiere, el ejemplo más claro tal vez sea el del antiguo compás situado ante las puertas de la catedral y antaño delimitado por un cancel gótico, restituido en un primer momento por Linazasoro mediante una serie de piedras colocadas verticalmente, luego sustituidas por unos sencillos bloques de caliza que sirven para sentarse ${ }^{34}$, semejantes a los empleados en otros lugares de la plaza (figuras 12 y 13). Esta modificación permitió, además, que el espacio del compás se abriera al antiguo recorrido del sacre en dirección al palacio de la Tau, incorporando la memoria de esa venerable ceremonia al nuevo diseño y generando continuidad histórica en el espacio.

33. LINAZASORO, José Ignacio, op. cit., supra, nota 9, p. 127. Una estrategia semejante se encuentra ya en Loyola, donde Linazasoro divide, para su ordenación, el entorno próximo del santuario en varios ámbitos diferentes: una plataforma, que contiene las lonjas de acceso y la plaza inmediata, y la zona ajardinada que llega hasta el río.

34. Ya el basamento de la escultura situada en la plaza de Azcoitia había sido empleado de manera similar. 
N24_ARQUITECTURAS AMPLIADAS

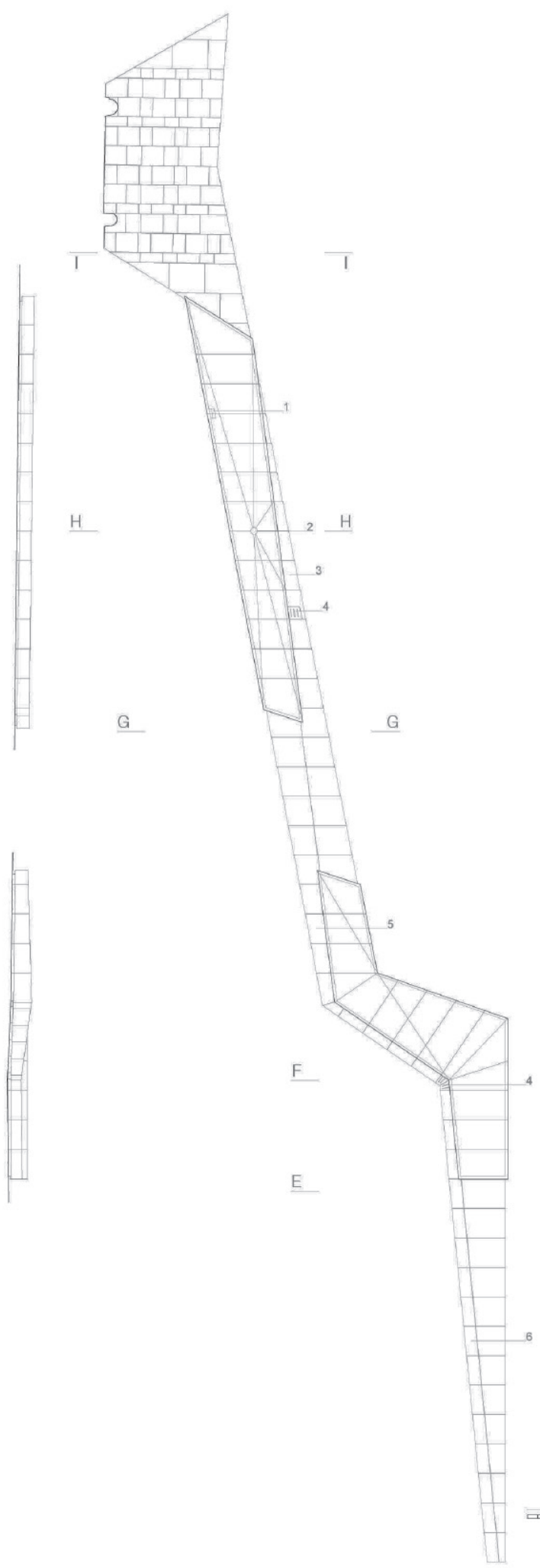

DETALLE DE BANCO Y FUENTE DEL PARVIS

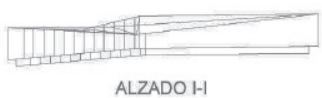

ALZADO H-1

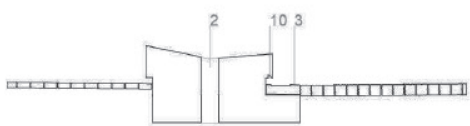

SECCIÓN H-H

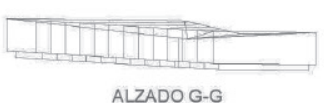

ALZADO G-G
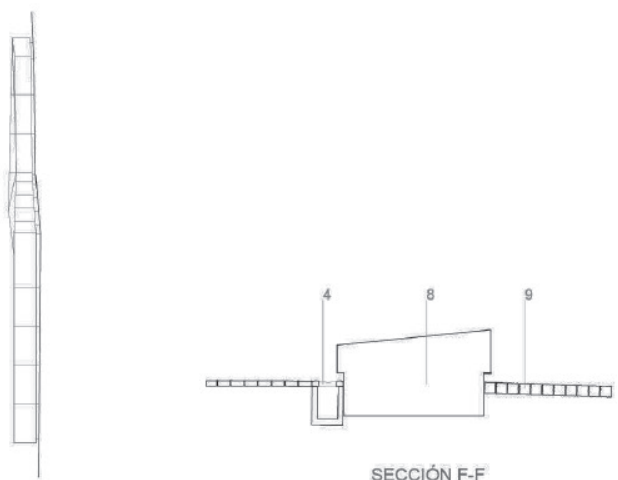

SECCIÓN F-F

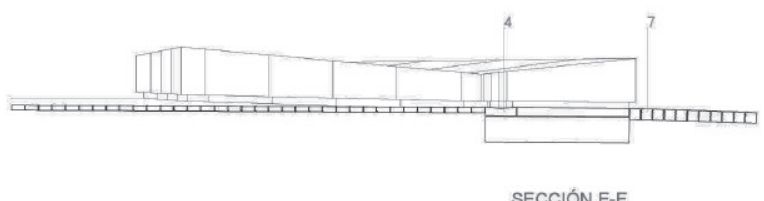

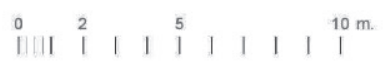


12. Detalle del elemento pétreo que define el nuevo parvis.

13. El nuevo parvis visto desde una torre de la catedral. Fotografía de Nicolas Waltefaulge.

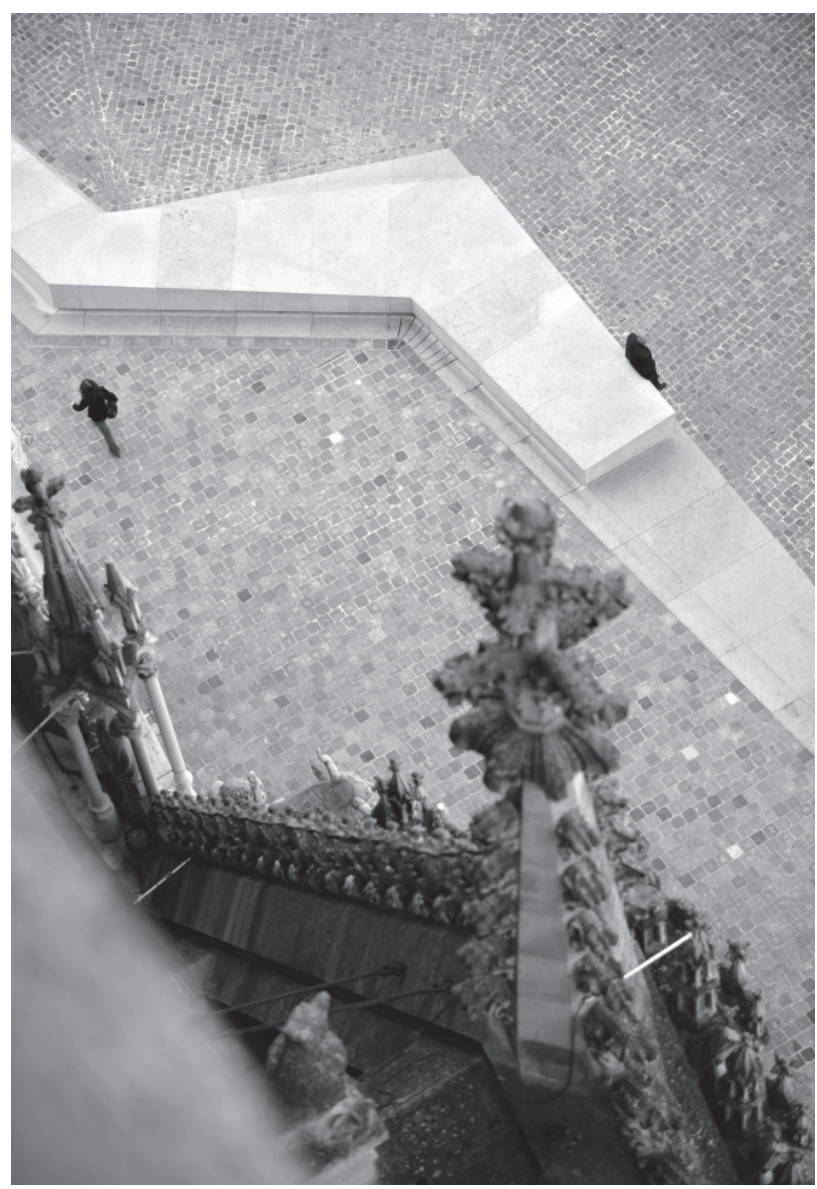

El resultado final de la actuación se consigue, pues, articulando tres criterios a los que Linazasoro también ha acudido en sus obras más recientes, donde las preexistencias juegan un importante papel: una certera integración de la propuesta en su entorno, una especial atención a los aspectos relacionados con la materialidad y un empeño en que su intervención pase desapercibida, asumiendo como propia esa vocación al anonimato que es característica de las mejores arquitecturas urbanas (figura 15). En el caso de Reims, todo se sustancia en la adecuada elección de los materiales y en el discreto diseño de los pavimentos que, frente a un cierto formalismo que había caracterizado

35. Curiosamente, esa referencia le llegó a Linazasoro a través de Utzon, quien la tuvo como un ejemplo canónico para algunas de sus soluciones proyectuales, según él mismo explicara en un célebre artículo: UTZON, Jørn. Platforms and Plateaus: Ideas of an Danish Architect. En: Zodiac, 1962, n. 10, pp. 114-117. No obstante, el tema de las plataformas no era nuevo en la obra del arquitecto donostiarra; tanto en Loyola como en Azcoitia había hecho uso de ellas, aunque no con el grado de sofisticación que encontramos en Reims.

36. LINAZASORO, José Ignacio, op. cit., supra, nota 4, p. 71.

37. GRIJALBA, Alberto; GRIJALBA, Julio. Conversando con José Ignacio Linazasoro. En: EGA. Expresión Gráfica Arquitectónica, 2020, n. ${ }^{\circ}$ 40, p. 25.

38. MONEO, Rafael. La soledad de los edificios. En: Fernando MÁRQUEZ; Richard LEVENE, eds. Rafael Moneo, 1967-2004. Antología de urgencia. El Escorial: El Croquis, 2004, p. 608. 
14. Sección de uno de los taludes, con banco pétreo y alcorque para la vegetación.

15. Formación de talud con encintado de piedra y

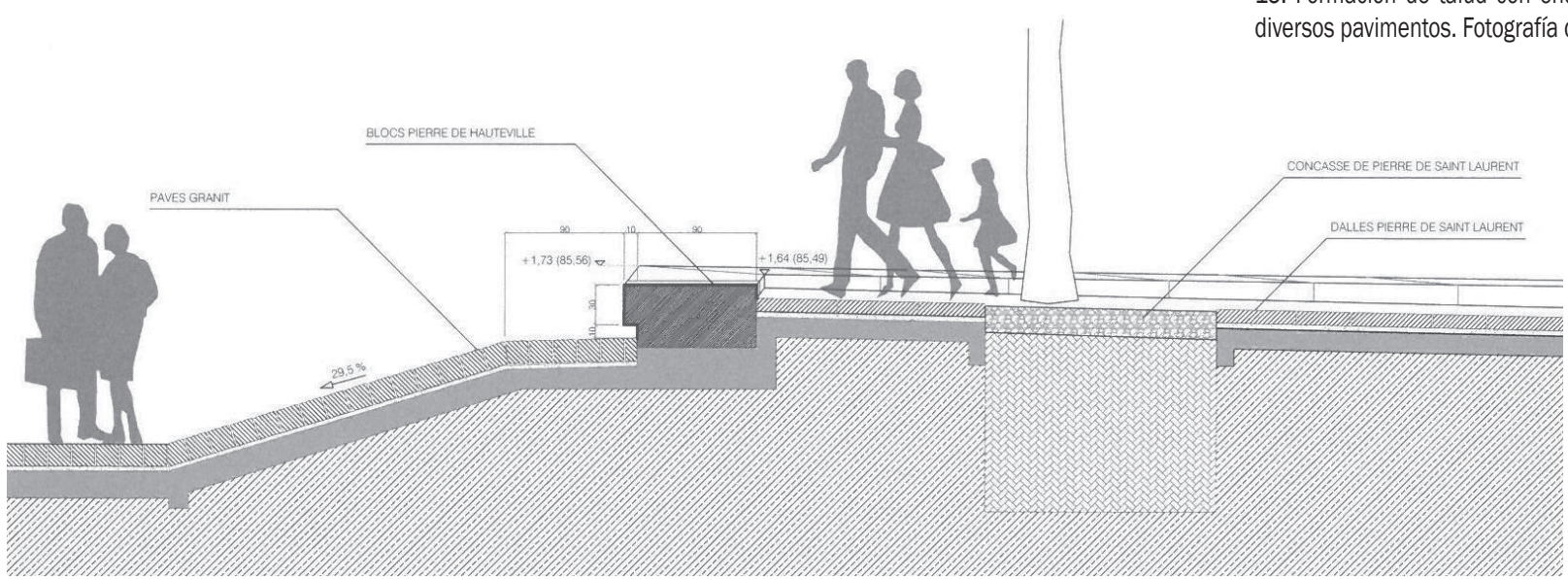

14

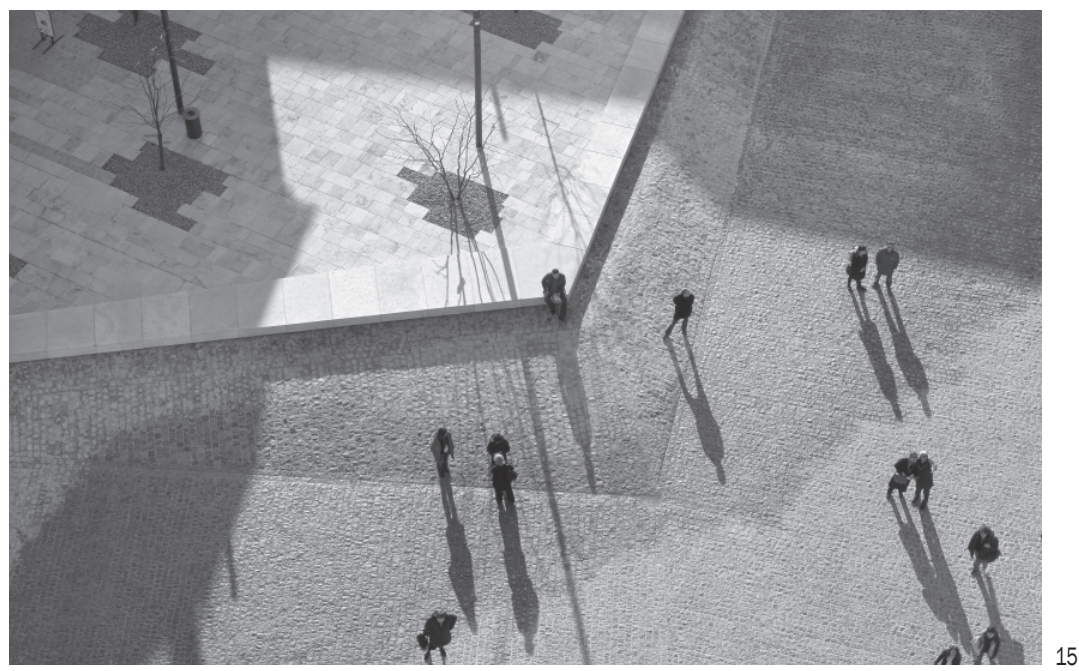

otras propuestas suyas para el espacio público ${ }^{39}$, contribuye a un protagonismo de la catedral acorde con su escala, que la sitúa a su vez en un contexto paisajístico atemporal y, por eso mismo, contemporáneo.

Esa contemporaneidad no es ajena a la tradición "antimoderna" que atraviesa la modernidad y que, como ha señalado Antoine Compagnon, "llega hasta nosotros"40. Es probablemente esta actitud la que ha permitido al arquitecto donostiarra resolver el viejo problema ya enunciado por Pierre Pinon cuando señalaba, a propósito de las catedrales góticas francesas, que "aislar un edificio es violar la historia"41. En este sentido, la plaza remense de Linazasoro puede ser considerada como una arquitectura expandida que forma parte de la catedral y la integra en la ciudad; tal vez por eso, la actuación haya sido aceptada por los usuarios con naturalidad y sin polémica. Queda, sin embargo, pendiente de resolver el entorno de la cabecera de la catedral, que sigue necesitado de un proyecto que termine de dar un tratamiento digno a ese extraordinario lugar de memoria que es Reims. $\square$

39. Pienso, por ejemplo, en la plaza de Agustín Lara en Madrid (1996-2001) o en el espacio interior de una manzana residencial en Baracaldo (2004-08). 40. COMPAGNON, Antoine. Los antimodernos. Barcelona: Acantilado, 2007, p. 239. "Yo creo -ha dicho Linazasoro- que la tradición es algo similar a los antimodernos de Compagnon: el mundo moderno, tomando lo mejor del pasado, vive en la tradición... que es mucho más que vivir sólo en la modernidad" (GUARRERA, Fabio, ed., op. cit., supra, nota 31, p. 22).

41. Con esas palabras titulaba su aportación al mencionado libro Places et monuments (JAKOVLEVIC, Nada; CULOT, Maurice, dirs., op. cit., supra, nota 12, p. 25). 


\section{Bibliografía citada}

BACKOUCHE, Isabelle. Expertiser la rénovation urbaine. Le cas de la France dans les années 1960. En: Genèses, 2008, n. ${ }^{\circ}$ 70, pp. 45-65.

BENDITO, María. La basílica devastada: el bombardeo de la catedral de Reims y la identidad nacional del arte gótico. En: Acta Artis. Estudis d'Art Modern, 2017, n. ${ }^{\circ} 4-5$, pp. 241-263.

BOUGEAULT, Bernard. La maison de verre d'Amiens. Son histoire vraie. París: Books on Demand, 2018.

CARRERO, Eduardo. La catedral habitada. Historia viva de un espacio arquitectónico. Barcelona: Universitat Autònoma de Barcelona, 2019.

COMPAGNON, Antoine. Los antimodernos. Barcelona: Acantilado, 2007.

DUBY, Georges. La época de las catedrales. Arte y sociedad, 980-1420. Madrid: Cátedra, 1997.

GRIJALBA, Alberto; GRIJALBA, Julio. Conversando con José Ignacio Linazasoro. En: EGA. Expresión Gráfica Arquitectónica, 2020, n. ${ }^{\circ}$ 40, pp. 16-39.

GUARRERA, Fabio, ed. Trentasette domande a José lǵnacio Linazasoro. Nápoles: CLEAN, 2014.

JAKOVLJEVIC, Nada; CULOT, Maurice, dirs. Places et monuments. Bruselas: Mardaga, 1984.

KRIER, Rob. Aménagement du Secteur Nord du centre de la ville d'Amiens. En: UR, 1985, n. ${ }^{\circ}$ 2, pp. 2-7.

LAURENT, Xavier. Grandeur et misère du patrimoine d'André Malraux à Jacques Duhamel (1959-1973). París: École des Chartes, 2003.

LE GOFF, Jacques. Reims, ville du sacre. En: Pierre NORA, ed. Les lieux de mémoire, vol. II-1: La Nation. París: Gallimard, 1986, pp. 89-194.

LINAZASORO, José Ignacio. El Escorial y la arquitectura del clasicismo. En: Carrer de la Ciutat, 1978, n. ${ }^{\circ}$ 5, pp. 5-10.

LINAZASORO, José lgnacio. El arte de la imitación en Juan de Villanueva. La Casita del Príncipe en El Escorial. En: Arquitectura, 1982, n. ${ }^{\circ} 239$, pp. 68-73.

LINAZASORO, José Ignacio. Cuatro proyectos. En: Arquitectura, 1983, n. ${ }^{\circ} 245$, pp. 53-63.

LINAZASORO, José Ignacio. Proyecto de ordenación del área del santuario de Loyola. En: Revista Internacional de Estudios Vascos, 1983, vol. 28, $n^{0} 2$, pp. 465-480.

LINAZASORO, José lgnacio. Textos críticos. Madrid: Asimétricas, 2017.

LINAZASORO, José Ignacio. Memoria de una búsqueda. Sobre escritos y proyectos. Valladolid: Universidad de Valladolid, 2019.

MARCHÁN FIZ, Simón. Una arquitectura con voluntad de adecuación. En: J. I. Linazasoro. Barcelona: Gustavo Gili, 1989, pp. 6-19.

MARTORELL, Josep; BOHIGAS, Oriol; MACKAY, David. La nouvelle Amiens. En: UR, 1985, n. ㄹ 2, pp. 10-15.

MOLEÓN, Pedro. El arquitecto Juan de Villanueva (1739-1811). Madrid: Akal, 2020.

MONEO, Rafael. Cuando la arquitectura quiere ser paisaje. En: Rocío PEÑA; Mario SANGALLI, eds. Luis Peña Ganchegui. Arquitecturas 1959-1994. San Sebastián: Fundación Kutxa \& Universidad del País Vasco, 1994, pp. 10-15.

MONEO, Rafael. La soledad de los edificios. En: Fernando MÁRQUEZ; Richard LEVENE, eds. Rafael Moneo, 1967-2004. Antología de urgencia. El Escorial: El Croquis, 2004, pp. 608-615.

MONEO, Rafael. La vida de los edificios. La mezquita de Córdoba, la lonja de Sevilla y un carmen en Granada. Barcelona: Acantilado, 2017.

NASCIMENTO OLIVEIRA, Francisco. Chão da cidade: permanencia e transformação. De metáfora a impressão digital da cidade. En: Proyecto, Progreso, Arquitectura, 2011, n. ${ }^{\circ}$ 4, pp. 138-151.

NAVARRO BALDEWEG, Juan. El tapiz, unas visiones y un proyecto. En: REIA. Revista Europea de Investigación en Arquitectura, 2021, n. ${ }^{\circ} 17, \mathrm{pp} .11-24$. ROSEMBERG, Muriel. Questions sur un conflit d'aménagement: le parvis de la cathédrale d'Amiens. En: Patrice MELÉ; Corinne LARRUE; Muriel ROSEMBERG, dirs. Conflits et territoires. Tours: Presses Universitaires François-Rabelais, 2004, pp. 165-186.

SOLÀ-MORALES, Ignasi de. Imitación esencial. La arquitectura de José Ignacio Linazasoro. En: Efrén GARCÍA GRINDA, ed. J. I. Linazasoro. Obras y proyectos, 1988-97. Madrid: Fundación Argentaria, 1998, pp. 9-13.

TRIGUEIROS, Luiz, ed. Fernando Távora. Lisboa: Blau, 1993.

UTZON, Jørn. Platforms and Plateaus: Ideas of a Danish Architect. En: Zodiac, 1962, n. 10, pp. 114-117.

Victoriano Sainz Gutiérrez (Madrid, 1961) es arquitecto por la Universidad de Sevilla, donde también se doctoró con una tesis sobre las ideas urbanísticas de Aldo Rossi. En la actualidad ejerce como Profesor Titular en la ETSA de Sevilla, de cuyo Departamento de Urbanística y Ordenación del Territorio ha sido director (2012-2020). Ha publicado, entre otros, los siguientes libros: La cultura urbana de la posmodernidad (1999), El proyecto urbano en España (2006), Aldo Rossi: la ciudad, la arquitectura, el pensamiento (2011) y Aldo Rossi y Sevilla. El significado de unos viajes (2019). 
LINAZASORO EN REIMS. EL ESPACIO PÚBLICO COMO MEMORIA DEL LUGAR LINAZASORO IN REIMS. THE PUBLIC SPACE AS THE MEMORY OF THE PLACE

Victoriano Sainz Gutiérrez (https://orcid.org/0000-0002-8125-5333)

p.35 Cathedrals are among the buildings that have historically suffered the most alterations to adapt their forms to new demands. They have often been shaped over time, acquiring a rich and complex life that has enabled distinguished examples of age-old architecture to arrive on our temporal doorstep integrated into more recent buildings: "Architecture that is well-defined-Moneo has written in this respect-will always be open to new interventions that indefinitely extend the life of a building" " 1 . To mention only one example, the complex organism of Canterbury Cathedral -the construction process of which lasted nearly ten centuries- springs to mind.

But today we know that these great architectural ensembles are inseparable from the urban environment into which they are inserted and which somehow completes them by enabling them to be perceived in a certain way. The life of buildings, then, has a deep connection with that of the city that houses them and, in the case of cathedrals, especially to the public spaces that surround them. These spaces have acted as the main scenario for numerous activities closely linked to their use since time immemorial, and it is true that "people did not enter the cathedral only to pray. There, the trade guilds and the entire community gathered for their civil meetings. [...] The business men thus considered the building as their own" 2 .

For this reason, given this organic relationship between the interior space of the church and the surrounding urban area, when we refer to the transformation processes to which the historical cathedrals were subjected, we must not forget the interventions that have been carried out in their surroundings. The successful integration of a building into its surroundings often depends on such interventions, particularly when many of the alterations carried out in recent times tended to treat the cathedral as a monument, isolating and making it independent of the urban fabric of which it used

p.36 to form an integral part ${ }^{3}$. In this context, intervention in the public space surrounding the cathedral becomes a crucial aspect of any modification and expansion project that seeks to provide the building with new architectural values. This article is dedicated to trying to illustrate it.

\section{J. I. LINAZASORO AND THE PUBLIC SPACE}

Among internationally recognised contemporary Spanish architects, José Ignacio Linazasoro is probably one of those who has devoted most attention to urban public spaces. In this vein, the project for reorganisation of the surroundings of Reims Cathedral (2003-2008) occupies a very special place in the work of the Basque architect, one that he described as "among the most difficult and interesting I have encountered" "4. Moreover, the building's location is among the most emblematic places in France since it was the scene of a ceremony of great historical significance: the country's monarchs were consecrated there (figure 1).

Linazasoro was awarded the contract to reform the square in front of Reims Cathedral as a result of winning two public competitions. The fact that there was a difference of ten years between the two competitions may go some way towards explaining why this is one of his most thoughtful and long-matured projects, to which must be added the experience accumulated by its author over the years in a series of projects of urban scale and nature, all approached with special attention to the pre-existing fabric and context. Not in vain was his architecture described by Marchán Fiz as "an architecture with a will to adapt" ${ }^{5}$, and the subsequent development of his work, focussed as it is on the organisation of public spaces, has only confirmed this. I will refer here only to those that I consider related in some way to the Reims project, omitting others that are also important but of less interest for the case in hand.

Nevertheless, it is important not to lose sight of the fact that this attention to public space has been a constant in Linazasoro's track record since his very beginnings; think, for example, of the projects of the 70's carried out with Miguel Garay: the reorganization of the old St. John's Square in Irún (1975), the first version of the Vergara cemetery (1976) or his proposal for the competition for a square in Legazpi (1979). In all of them, with a neo-rationalist language that sprung from the enthrallment that the Tendenza produced in many of the young architects of that time, some features already appeared that will be central in his later projects: the interest in empty space, the emphasis on the design of the pavements and attention to the surrounding architecture as strategies to give meaning to the place.

But there are two projects above all, very different from each other, both drawn up in 1982 by Linazasoro (now working alone) that may have been a kind of laboratory to experiment with some of the fundamental ideas that would

p.37 enable him to shape the intervention in Reims: reform of the forecourt of the Sanctuary of Loyola and refurbishment of the Balda Square in Azcoitia (Gipuzkoa, Basque Country). He believes they should be seen as "the statement of a different modernity not identified with that derived from the avant-garde, and from which the reuse of a whole range of forms and design instruments inherited from tradition is proposed [...] which, far from being obsolete, are finally the most suitable for recovery of an architectural image [... . far removed from all experimentalism" ${ }^{6}$. Those were the years when he published his book El proyecto clásico de la arquitectura (1981) and recovered forms rooted in history.

This is probably where Linazasoro's interest in working with pre-existing assets comes from: he considers that the problems we are facing have already been faced by others and seeks a reference for the project in their solutions ${ }^{7}$. We are dealing -in the case of his project for the Sanctuary of Loyola- with a proposal that tries not only to adapt Carlo 
Fontana's building to an environment that was largely foreign to him, but above all, dovetail it into the surroundings in accordance with premises already contained in the original plans. From this perspective, and taking the "urban" character of the sanctuary's architecture into account, it was first of all a matter of resolving its relationship with the grounds. The platform initially projected by Linazasoro as support for the historical complex was later reduced to two smaller symmetrical raised volumes the function of which was to resolve the intersection of the monumental stairway with the space located immediately in front of the building (figure 2).

Having recourse to stone paving, the composition of which is meticulously designed, clearly anticipates what would become a constant in Linazasoro's successive interventions on public spaces in which materiality and vegetation are the basic components to ensure the timelessness and anonymity of the finally chosen solution. This is the crucial aspect of this project, despite the fact that the language is still vaguely classicist, which is also evident in p.38 the first version of the Balda Square, where the idea of order contained in both the repetition of the arches and in the regularity of the grid-patterned paving is also an echo of a figurative architecture still focused on compositional issues linked to classicism (figure 3); more specifically, on the platform that serves as a support for placement of El Escorial, a question that the architect had already dealt with "from a theoretical point of view" in a previous article 8 .

Furthermore, there was another pre-existing question latent in both projects that not only provides the key to the argument we intend to develop here, but to which Linazasoro would return later, namely, the concept of public space as an "expanded architecture" in the sense that his project integrates certain architectures immediately related to it into an overarching unit. In Loyola, the terrace and the garden that Linazasoro designed -but which were not executed-in front of the sanctuary were intended to "continue the idea that would derive from the building's own construction plans" ${ }^{9}$, just as Fontana had conceived them. In Azcoitia, however, it was a question of enabling a more fluid and articulated p.39 dialogue between the Balda and Irízar family mansions through an architecture that solved the problems deriving from the complex topography of the surroundings at the same time as it constructed the plaza.

A similar design strategy can be found in a historical intervention that Linazasoro was studying at that time: the Casita del Príncipe, designed by Juan de Villanueva in the vicinity of El Escorial ${ }^{10}$. It is a small recreational pavilion composed of an initial volume built between 1771 and 1775 and later extended to the west with a "hall, garden extension and pond" between 1781 and 1785. The recreational purpose of the building required a close relationship between the new interior space and the back garden, which were executed simultaneously. In fact, the reason for the extension was probably the inadequacy of the small rooms available in the front part, and the lack of a suitable place for recreation half way between the pavilion and the surrounding oak forest. The ensemble "maintains an unaltered superior order, a compositional procedure that finally acquires greater richness and complexity as it becomes more expressive by manifesting its content with greater clarity to the outside" 11.

\section{REIMS, PLACE OF MEMORY}

While engaged in these projects for Loyola and Azcoitia, Linazasoro also took care, as part of his pedagogic mission, of the surroundings of the Notre-Dame de Paris Cathedral by setting his students an exercise centred on reordering the urban emptiness surrounding the building. This work led him to thoroughly explore the dual intervention performed in the 19th century: on the one hand, the "restoration" of the cathedral proposed by Viollet-le-Duc following the rationality of certain stylistic principles that would supposedly rejuvenate its image and, on the other, the destruction of the surrounding medieval fabric in order to configure it as an isolated monument. And all this with the aim of staging the great national narrative that converted the Gothic cathedrals into symbols of the unity of the French people. Thus, Linazasoro was also able to familiarise himself with the debates that took place in France during the second half of the 20th century concerning the options for reshaping the vicinity of these spaces that surrounded the great cathedrals ${ }^{12}$.

With this knowledge Linazasoro had to face his first French project after becoming the successful tenderer of a call for ideas, announced in 1991, to reorganise the surroundings of the Reims Cathedral ${ }^{13}$. The city was not only a significant lieu de mémoire for the French nation but the essential purpose of the competition was related to the main of its monuments: the Gothic cathedral. In the case of Reims since both the city and especially the cathedral were associated, in the memory of the French people, with an extremely particular ceremony: the consecration of their monarchs, celebrated there since the time of the Carolingians ${ }^{14}$. Nevertheless, with the passage of time the cathedral's surroundings had undergone significant changes. An urban space had been shaped on the basis of axiality and detachment, breaking the organic relationship that had historically existed between the cathedral and its surroundings.

The first changes in the medieval fabric of Reims took place as a result of the embellissement plan designed in p.40 1754 by the engineer Jean-Gabriel Legendre (figure 4). It was already suggested the option of opening up a square in front of the Cathedral. In the $19^{\text {th }}$ century, the Libergier street was opened up as a perspective axis that connected the Cathedral with the canal (figure 5), and Hôtel-Dieu, a former hospital, to be replaced by the current Palais de 
Justice, thus expanding the forecourt space. But the great transformation of the surrounding area was due in large part to the bombing suffered by the city during World War I, as a result of which a good proportion of the buildings that surrounded the cathedral was destroyed ${ }^{15}$. The reconstruction plan devised in the 1920s, conceived as a continuation of the $19^{\text {th }}$ century approaches, left the apse of the Cathedral in plain view.

In the context of the reflection that took place in France as early as the 1960's on the question of the surroundings of heritage buildings ${ }^{16}$, there was an awareness of the need to intervene in those of the Reims Cathedral, although the criteria for putting the concept into practice were not settled until the mid-eighties within the framework of the refurbishment of the historical centre of the city. The debates throughout the country focused on whether re-occupying the voids that surrounded the cathedrals with buildings was a good idea or not and on the most suitable architecture with which to do so if it were. There were powerful citizen protests in Reims in the mid-seventies against the plan to construct a concrete and glass building opposite the Cathedral to house the Court of Appeal, which was finally located in a less compromised place from the heritage point of view. This was not an isolated case, as something similar happened in Rouen and Amiens.

p.41 The conflict in Amiens may exemplify the debates that took place in France in those years, although in this case it was focused more on the architecture of the buildings around the cathedral than on the public space itself. The controversy began in the early seventies with the construction of the building known as the maison de verre, a supposedly Miesian building located in the vicinity of the cathedral ${ }^{17}$. In the following decade a restricted competition, won by Rob Krier, was called to reorder the area around the cathedral (figure 6). With a purely morphological approach, the Luxembourgian architect proposed to recover the scale of the medieval city by constructing a series of historicist structures that formed a series of concatenated public spaces, thus reconfiguring the structure of the cathedral forecourt ${ }^{18}$. The proposal was never put into practice and the French architect Bernard Huet took charge of the planning, erecting, in the late 1990s, a building in front of the cathedral that hid the pre-existing glass building from view ${ }^{19}$.

Linazasoro, who was familiar with these solutions, would choose a different approach for his Reims project in which the intervention on the urban space would play the major role. Neither Krier's nor Huet's proposals convinced him. In his opinion, other architectures were not required to solve the problem of the void that had arisen around the cathedral. What he proposed in Reims, in fact, was to redefine the shape of the square without trying to change its size. He considered that the proposals for Amiens went "against the grain of a process which, although had had a somewhat negative effect, was already irreversible since it was not logical to reduce the dimensions of a public space that had been achieved by demolition of the old buildings" ${ }^{20}$. His ideas were, therefore, closer to the landscaping approach of Oriol Bohigas's project for Amiens, which revolved around treatment of the void as part of a network of free spaces to be defined by the intervention ${ }^{21}$. The fact that Krier won that competition shows which were the dominant ideas in this respect in France at the time.

Linazasoro's goal in Reims was clear: "It was all about recovering the lost scale and, without trying to undertake the impossible task of reconstructing the missing urban fabric, designing an environment which, by means of a new layout and a more appropriate geometry, would enable us to recover the old balance between the monument and the city" 22. Consequently, the forecourt is once again understood as an integral part of the monument, which was part of the place. It was conceived to contribute to relocating the Cathedral in an environment capable of enabling it to be perceived more in line with the experience of the medieval city from which it had arisen. Perhaps that is why Linazasoro said that his proposal for this public space was designed so that "it could serve as a paradigm for other types of similar

p.42 intervention in France itself" 23

\section{A CONTEMPORARY PROJECT FOR THE CATHEDRAL SQUARE}

Linazasoro travelled to the Nordic countries during the first half of the decade of the 90s and visited certain works by architects like Aalto, Asplund and Lewerentz, whose work he had always liked. First-hand knowledge of Jyväskyla University, the experimental house in Muraatsalö or the Stockholm South Cemetery, to name only a few that have a bearing on our argument, led him to delve more deeply into a series of questions that he had already dealt with but that would begin to influence his projects in a new way. I am referring, among other things, to the importance of materiality, the dialogue between the interior and the exterior of buildings or the way they are lodged in the place. With respect to the architecture of this group of masters, he was mainly interested in "the urban, fragmentary character and the materiality of their work and in that sense, much more than its forms, [he was interested in] their references, which were related some of the permanent interests of [his] work, such as historic cities and the urban project" ${ }^{24}$.

In this context, the finally constructed version of Azcoitia's project (1997-1999) would undergo a substantial simplification, which well expresses this renewed approach to Linazasoro's architecture. The leading role of preexisting structures is underlined here by the anonymous, discreet elements that the project introduced: the paving, less rigid and more contextual; the wall, clearly defined by its materiality; the stone base that works both as a bench

p.43 and as the support for Oteiza's sculpture (figure 7). Thus, a distinctly contemporary space is created in which the emphasis is placed on the abstract geometry of the layout and on the materials used in its construction. "At that time I was thinking -the architect has said-about the two squares by Luis Peña in San Sebastian, especially Trinity Square, which I saw as an excellent example of the search for anonymity and timelessness which, except in very rare cases, characterise public spaces" ${ }^{25}$. 
Indeed, Plaza de la Trinidad (1963) is a clear example of how to integrate and articulate, through public space, a formless group of architectural elements of very different kinds -including the pre-existing handball (frontón) walllocated in a topographically difficult and physically degraded context. By having recourse to materiality, Peña managed to "achieve unity and harmony with a chorus of very diverse voices [...], showing that the practice of architecture is also establishing a dialogue with what was built in the past, turning it into a tangible reality and endowing elements that had lost all meaning with new significance" 26 .

The four squares in the historic and cultural centre designed by Fernando Távora (1987-1995) in the Portuguese city of Guimarães are also conceived along the same lines. In this case, paving stones and small urban furniture components are also employed, giving rise to a different way of composing and organising the public space that responds to the different character of the architecture of each square, which in a certain sense it extends and interprets. "Thus, the character of the Praça do Município is Baroque, that of Praça de Santiago Medieval, Renaissance that of the Largo João Franco and Romantic that of the Largo Condessa do Juncal. Different personalities inserted into an urban route that guarantees its unity within its diversity" 27.

The decidedly contemporary expression of the language adopted by both architects and the emphasis they place on the paving treatment are not a long way from the solution adopted by Linazasoro in Azcoitia, where the paving is designed as a "metaphor full of meanings that is assumed as much for the spatiality it institutes as for the urbanity that it intuits" ${ }^{28}$. In all these cases, as in Reims Cathedral square, the paving becomes the tapestry where the old threads p.45 of pre-existing architectures are woven with other completely new ones, thus legitimizing the creative decisions made in the design of the pavements the materiality of which appears as an expression of the passage of time. As Navarro Baldeweg has stated in this regard, it is clear that "the goals of any project are not met, or are not only met, by the beauty of an object, but also by the functional capacity of bringing together essential sensations and emotions of a previous life" 29 .

In the case of Reims Linazasoro had to seek out this "previous" life in the history of the city. The urban approach of his proposal was required by the terms of the first ideas competition for the Cathedral surroundings called in 1992. The Basque architect proposed an overall reconsideration that endows the void that precedes the great Gothic façade with new meaning, recovering the values of the lost medieval heritage but with a clearly contemporary attitude. His intention was to bring the Cathedral out of its isolation (figure 8). To do so, he changed the orientation of the forecourt space and opened it up to new urban axes that enabled the appearance of oblique views of the Cathedral. The design of the forecourt follows in the footsteps of Camillo Sitte on the basis of the lessons learned from the piazzas of historic Italian cities, reinterpreted in a contemporary key.

Sitte's analyses already occupied an important place among the references in Places et Monuments ${ }^{30}$. In particular, it is easy to identify the images of the Piazza del Campo in Siena or of the Piazza della Signoria in Florence, featuring therein as the source of certain design decisions by Linazasoro. Among them, it is worth noting the emphasis placed on the topography and the drawing of the paving, as well as the shifting of the statue of Joan of Arc to place it in a position that enables it to articulate the different spatial sequences contained in the proposal. But it is above all in the discourse on the void where the influence of the Viennese architect is decisive. Linazasoro said in this regard: "Sitte shows that space, made with few well-located things and completely flexible from a functional point of view, is essential in old cities. This is precisely what I had in mind when I designed the Reims Square" ${ }^{31}$.

The 1992 competition sought to establish a frame of reference for subsequent specific interventions in the area of influence of the Cathedral. In this context, in 1997 another competition was announced, this time to design the media library to be built at the entrance to rue Rockefeller on the site occupied by the former police station. Linazasoro, in conjunction with the Thiénot-Ballan studio, submitted a project which, while it failed to win the competition, nevertheless enabled him to continue reflecting on the place. The competition to define the square in front of the Cathedral was announced in 2003, and once again Linazasoro presented a project with the same studio. This time, they submitted the winning project. The original project was then reformulated and updated, producing "a more essential version, with a clearer but always irregular geometry to avoid competing with the geometry of the Cathedral" 32 (figures 9 and 10).

The idea was to return the Cathedral to an environment the scale of which was adapted to that of the old medieval plot organisation, now recovered by a project strategy combining slopes and vegetation to recall its previous structure by analogy. To do so, he created a subtle artificial topography intended to endow the public space with the shape of the preceding urban environment using a geometry similar to that of the medieval layout (figure 11). Four clearly differentiated spaces were formed: "a central space represented by the square itself, two lateral spaces in the form p.47 of a platform that evoke, more or less literally, the shape of the missing blocks -each with a different treatment- and a forecourt or sagrato next to the façade that recovers a space lost in the 18th century" ${ }^{33}$. The entire operation aims to recreate an apocryphal past as if it were an archaeological permanence.

The project was simplified somewhat during the execution phase, unifying the expressive resources intended to create the different spatial areas into which the pre-existing inhospitable void was divided. As far as materiality is concerned, the clearest example is perhaps the old compass located in front of the Cathedral doors and once delimited by a Gothic cancellus. Linazasoro restored it at first by means of a series of vertically-arranged stones which were then replaced by a few simple blocks of limestone that also serve as seating ${ }^{34}$, similar to those found in other locations in the square. This modification also enabled the space around the compass to open up to the old Sacre or 
anointing route, in the direction of the Palace of Tau, thus incorporating the memory of that venerable ceremony into the new design and generating historical continuity in the space.

p.49 The construction of a new topography is probably the most decisive commitment of the project. Taking as a starting point the platforms proposed in the 2003 competition, which were strongly influenced by those of the preHispanic Monte Albán complex near Oaxaca in Mexico ${ }^{35}$, the final shape of these platforms evolved into less abrupt, more nuanced and accessible forms. The different types of paving were crucial to define them and, as in Loyola and Azcoitia, constituted one of the central arguments of the entire proposal. In this case, they seek to establish "a balance between the mineral and the vegetal that serves as the scenic background to what we could call the central space: the square itself" ${ }^{36}$. The plant masses were also simplified and naturalised with respect to the initial, more "architectonic" proposal: the French-cut linden trees were replaced by various species that have been allowed to grow freely.

This attitude, linked less to geometry and more sensitive to a certain naturalness of things, is the result of the passage of time. Nevertheless, it is closely related to the construction experience (figure 14). At Loyola, for example, Linazasoro had planned to use conventional slabs, but "the contractor -he later recalled-suggested changing to another hand-crafted kind with a beautiful irregular finish. Their cut and texture were so much more interesting than what I had planned" ${ }^{37}$. This reminds us that the rigor of the drawing only reaches its full definition by passing the test of physical materialisation. On this point the Basque architect shares the thoughts of many Spanish colleagues of his generation. As Moneo said, "architecture becomes what it really is by accepting and agreeing with the limitations and restrictions, with what construction implies" 38 .

The final result of the intervention is achieved thanks to the articulation of three criteria on which Linazasoro has relied in a good part of his most recent works, in which pre-existing elements play an important role: an impeccable integration of the proposal into its surroundings, special attention to aspects related to materiality while endeavouring, at the same time, to ensure that his intervention goes unnoticed. He takes the vocation of anonymity characteristic of the best urban architectures as his guiding principle (figure 15). In the case of Reims, everything is grounded in the suitable choice of materials and in the discreet design of the paving which, in contrast to a certain formalism that had

p.50 characterised his other proposals for public spaces ${ }^{39}$, emphasizes the Cathedral in accordance with its scale, placing it in a timeless and, for that very reason, thoroughly contemporary landscape context.

This contemporaneity is not foreign to the "anti-modern" tradition that forms a current within modernity and which, as Antoine Compagnon pointed out, "continues to this day" ${ }^{40}$. It is probably this attitude that has enabled Linazasoro to solve the old problem already enunciated by Pierre Pinon when he pointed out, with respect to the French Gothic cathedrals, that "isolating a building is to violate history" ${ }^{41}$. Consequently, Linazasoro's square in Reims can be considered to constitute expanded architecture that forms part of the Cathedral and integrates it into the city. Perhaps this is why his solution has been accepted by the city's inhabitants naturally and without controversy. However, the area around the apse of the Cathedral has yet to be resolved and is still in need of a project that will end up endowing that extraordinary place of memory called Reims.

1. MONEO, Rafael. La vida de los edificios. La mezquita de Córdoba, la lonja de Sevilla y un carmen en Granada. Barcelona: Acantilado, 2017, p. 46.

2. DUBY, Georges. La época de las catedrales. Arte y sociedad, 980-1420. Madrid: Cátedra, 1997, p. 115. There is more detailed information in CARRERO, Eduardo. La Catedral Habitada, Historia Viva de un Espacio Arquitectónico. Barcelona: Universitat Autònoma de Barcelona, 2019.

3. In the Spanish context, the isolationist works around the cathedrals of León and Burgos should not be forgotten. Although they were performed in the early 20th century, they were conceived some decades earlier in the wake of what had already been done to so many French cathedrals.

4. LINAZASORO, José Ignacio. Memoria de una búsqueda. Sobre escritos y proyectos. Valladolid: Escuela Técnica Superior de Arquitectura de Valladolid, 2019, p. 71.

5. MARCHÁN FIZ, Simón. Una arquitectura con voluntad de adecuación. In: J. I. Linazasoro. Barcelona: Gustavo Gili, 1989, pp. 6-19.

6. LINAZASORO, José Ignacio. Cuatro proyectos. In: Arquitectura, 1983, no. 245, p. 53.

7. This "negotiation with what has already been built" is carried out in relation "to the architecture in its history as richness and heritage, as the scope of action in which the production of new architecture finds a privileged situation of continuity and stimulus" (SOLÀ- MORALES, Ignasi de. Imitación esencial. La arquitectura de José lgnacio Linazasoro. In: Efrén GARCÍA GRINDA, ed. J. I. Linazasoro. Obras y proyectos, 1988-97. Madrid: Fundación Argentaria, 1998, p. 10).

8. LINAZASORO, José Ignacio, op. cit., supra, note 6, p. 54; the mentioned article is LINAZASORO, José Ignacio. El Escorial y la arquitectura del clasicismo. In: Carrer de la Ciutat, 1978, no. 5, pp. 5-10.

9. LINAZASORO, José Ignacio. Proyecto de ordenación del área del santuario de Loyola. In: Revista Internacional de Estudios Vascos, 1983, vol. 28, no. 2, p. 472.

10. LINAZASORO, José Ignacio, El arte de la imitación en Juan de Villanueva. La Casita del Príncipe en El Escorial. In: Arquitectura, 1982, num. 239, pp. 68-73. The article focusses on issues related to the classical tradition around which Linazasoro's research revolved at the time, but it is easy to imagine that he must have been especially interested in the refined holistic approach and its brilliant solution to the integration of the interior and the exterior.

11. MOLEÓN, Pedro. El arquitecto Juan de Villanueva (1739-1811). Madrid: Akal, 2020, p. 13.

12. To verify this, we only need to review the interventions compiled in JAKOVLEVIC, Nada; CULOT, Maurice, ed. Places et monuments. Brussels: Mardaga, 1984, including Linazasoro's proposal for the square in Azcoitia (pp. 78-81) and two of the projects drawn up by his students of the San Sebastian School of Architecture (pp. 158-163).

13. ${ }^{13}$ In addition to the Reims cathedral square, Linazasoro has designed a conference centre in Troyes in France (2010-2014) and, more recently, he won the design competitions for the surroundings of the basilicas of Notre-Dame-du-Port in Clermont-Ferrand (2018) and Notre-Dame-des-Miracles in Mauriac (2019).

14. See in this respect: LE GOFF, Jacques. Reims, Ville du Sacre. In: NORA, Pierre, ed. Les lieux de mémoire, vol. II-1: La Nation. Paris: Gallimard, 1986, pp. 89-194.

15. See BENDITO, María. La basílica devastada: el bombardeo de la catedral de Reims y la identidad nacional del arte gótico. In: Acta Artis. Estudis d'Art Modern, 2017 , no. 4-5, pp. 241-263. 
16. On the activities of the Commission des Abords at that time see BACKOUCHE, Isabelle. Expertiser la rénovation urbaine. Le cas de la France dans les années 1960. In: Genèses, 2008, no. 70, pp. 45-65. For the context of the debates on heritage buildings see LAURENT, Xavier. Grandeur et misère du patrimoine d'André Malraux à Jacques Duhamel (1959-1973). Paris: École des Chartes, 2003, especially pp. 147-162, devoted to the question of the abords.

17. The point of view of the project architect can be perused in BOUGEAULT, Bernard. The maison de verre d'Amiens. Son histoire vraie. Paris: Books on Demand, 2018.

18. See KRIER, Rob. Aménagement du Secteur Nord du centre de la ville d'Amiens. In: UR, 1985, no. 2, pp. 2-7.

19. There is a summary of the discussions on this issue in ROSEMBERG, Muriel. Questions sur un conflit d'aménagement: le parvis de la cathédrale d'Amiens. In: MELÉ, Patrice; LARRUE, Corinne; ROSEMBERG, Muriel, dirs. Conflits et territoires. Tours: Presses Universitaires François-Rabelais, 2004, pp. 165-186.

20. LINAZASORO, José Ignacio, op. cit., supra, note 4, p. 54.

21. See MARTORELL, Josep; BOHIGAS, Oriol; MACKAY, David. La nouvelle Amiens. In: UR, 1985, no. 2, pp. 10-15.

22. LINAZASORO, José Ignacio. Textos críticos. Madrid: Asimétricas, 2017, pp. 111-112.

23. LINAZASORO, José Ignacio, op. cit., supra, note 4, p. 69.

24. LINAZASORO, José Ignacio, op. cit., supra, note 4, p. 53.

25. LINAZASORO, José Ignacio, op. cit., supra, note 4, p. 55.

26. MONEO, Rafael. Cuando la arquitectura quiere ser paisaje. In: Rocío PEÑA; Mario SANGALLI, eds. Luis Peña Ganchegui. Arquitecturas 1959-1994. San Sebastián: Fundación Kutxa-Universidad del País Vasco, 1994, p. 12.

27. TÁVORA, Fernando. Rehabilitações urbanas. Guimarães. In: Luiz TRIGUEIROS, ed. Fernando Távora. Lisbon: Blau, 1993, p. 178.

28. NASCIMENTO OLIVEIRA, Francisco. Chão da cidade: permanencia e transformação. De metáfora a impressão digital da cidade. In: Proyecto, Progreso, Arquitectura, 2011, no. 4, p. 140.

29. NAVARRO BALDEWEG, Juan. El tapiz, unas visiones y un proyecto. In: REIA. Revista Europea de Investigación en Arquitectura, 2021, no. 17, 2021, p. 13. The term "tapestry" is equivalent to "context". The importance granted to context here is closely aligned to what Linazasoro was seeking in his work, although the accents are certainly different. 30. JAKOVLEVIC, Nada; CULOT, Maurice, dirs., op. cit., supra, note 12, pp. 47-55, contains the first chapter of the well-known treatise of this Austrian urban planner, with illustrations from the first French edition of 1918.

31. GUARRERA, Fabio, ed. Trentasette domande a José Ignacio Linazasoro. Naples: CLEAN, 2014, p. 39

32. LINAZASORO, José Ignacio, op. cit., supra, note 9, p. 69.

33. LINAZASORO, José Ignacio, op. cit., supra, note 9, p. 127. A similar strategy is already found at Loyola where Linazasoro divides the surroundings of the sanctuary into several different areas in order to arrange them: a platform which contains the raised volumes and the immediate square, and the garden area that extends down to the river. 34. The base of the sculpture located in the Balda Square, in Azcoitia, had already been treated in a similar way.

35. Interestingly, this reference came to Linazasoro through Utzon, who held it as a canonical example in some of his architectural solutions as he himself explained in a famous article: UTZON, Jørn. Platforms and Plateaus: Ideas of a Danish Architect. In: Zodiac, no. 10, 1962, pp. 114-117. Notwithstanding the above, the issue of platforms was not new in Linazasoro's work. He made use of them at both Loyola and Azcoitia, although not with the degree of sophistication that we find in Reims.

36. LINAZASORO, José Ignacio, op. cit., supra, note 4, p. 71.

37. GRIJALBA, Alberto; GRIJALBA, Julio. Conversando con José Ignacio Linazasoro. In: EGA. Expresión Gráfica Arquitectónica, 2020, no. 40, 2020 , p. 25.

38. MONEO, Rafael. La soledad de los edificios. In: Fernando MÁRQQUEZ; Richard LEVENE, eds. Rafael Moneo, 1967-2004. Antología de urgéencia. El Escorial: El Croquis, 2004 , p. 608.

39. It brings to mind, for example, the Agustín Lara Square in Madrid (1996-2001) or the interior of a residential block in Baracaldo (2004-2008).

40. COMPAGNON, Antoine. Los antimodernos. Barcelona: Acantilado, 2007, p. 239. "I believe -Linazasoro has said- that tradition is something similar to Compagnon's anti-moderns: the modern world, taking the best of the past, lives in tradition... which is much more than just living in modernity" (GUARRERA, Fabio, ed., op. cit., supra, note 31, p. 22).

41. These words preceded his contribution to the aforesaid book Places et Monuments (JAKOVLEVIC, Nada; CULOT, Maurice, dirs., op. cit., supra, note 12, p. 33). 


\section{Autor imagen y fuente bibliográfica de procedencia}

Información facilitada por los autores de los artículos:

página 19, 1. WOODBRIDGE, Kenneth. Princely Gardens. Nueva York: Rizzoli, 1986; página 21, 2. ALPHAND, J. C. Adolphe. Les Promenades de Paris. Princeton: Princeton Architectural Press, 1984, p. 199; página 22, 3. AURICOSTE, Isabelle; TONKA, Hubert. Parc Ville Villette. Seyssel: Champ Vallon, 1987; páginas 23,24 y 25, 4, 5 y 6. Fotografías de Ramón R. Llera; páginas 26 y 27, 7 y 8 . BASSO PERESSUT, Luca; CALIARI, Pier Federico, eds. Piranesi Prix de Rome. Progetti per la nuova Via dei Fori Imperiali. Roma: Aión Edizioni, 2017; página 28, 9. LAB/PAP Laboratorio de Paisaje Arquitectónico, Patrimonial y Cultural; páginas 28 y 29, 10, 11 y 12 . BASSO PERESSUT, Luca; CALIARI, Pier Federico, eds. Piranesi Prix de Rome. Progetti per la nuova Via dei Fori Imperiali. Roma: Aión Edizioni, 2017; páginas 30 y 31 ,13, 14 y 15. ÁLVAREZ, Darío; IGLESIA, Miguel Ángel de la. Modelos de paisajes patrimoniales. Estrategias de protección e intervención arquitectónica. Valladolid: LAB/PAP y Departamento de Teoría de la Arquitectura y Proyectos Arquitectónicos, Universidad de Valladolid, 2017; página 36, 1. Philippe BOURDIN, “Le sacre de Louis XV", Histoire par l'image [en línea], consultado el 20 de agosto de 2020. Disponible en: http://histoire-image.org/fr/etudes/sacre-louis-xv; página 37, 2. Archivo de José lgnacio Linazasoro Rodríguez; página 38, 3. JAKOVUEVIC, Nada; CULOT, Maurice, dirs. Places et monuments. Bruselas: Mardaga, 1984; página 40, 4. RIGAUD, Olivier. Les plans anciens de Reims, 16001825. Dossier iconographique. Reims: RHA, 1991; página 41, 5. Biblioteca Municipal de Reims [en línea], consultado el 20 de agosto de 2020. Disponible en: https:// commons.wikimedia.org/wiki/File:Rue_Libergier_Cathédrale_Reims_Varin_30131.jpg; página 42, 6. Revista UR, 1985, n. ${ }^{\circ}$ 2; página 43, 44, 46, 47, 48, 49, 50, 7, 8, 9 , 10, 11, 12, 13, 14, 15. Archivo de José Ignacio Linazasoro Rodríguez; página 54, 1. Eisenman Architects Archives; página 55, 2. DR2004:0360:003 Cedric Price fonds Canadian Centre for Architecture; página 56, 3. DR2004:0355:007. Cedric Price fonds Canadian Centre for Architecture; página 57, 4. DR2004:0355:006. Cedric Price fonds. Canadian Centre for Architecture; página 59, 5. ARCH267828. IFCCA Prize Competition for the Design of Cities fonds Canadian Centre for Architecture; página 59, 6. Dominio público.; página 60, 7. Archivo Lacaton \& Vassal; página 61, 8. Fotomontaje de los autores a partir de Google Street View (2018); página 62, 9. Fotografía de Philippe Ruault:; página 63, 10. Muestra de la web del proyecto con el reportaje fotográfico de Philippe Ruault.; página 64,11 . Archivo Lacaton \& Vassal.; página 65,12 . Vista aérea en Google Maps (2018):; página 65, 13. Vista obtenida en Google Street View (2018); página 66, 14. Archivo Lacaton \& Vassal, 2004.; página 67, 15. Vista obtenida a través de Google Street View (2018); páginas 74 y 75, 1, 2 y 3. @ Krijn de Koning, cortesía del artista; páginas 76 y 77, 4, 5 y 6 . @ Jessica Stockholder, cortesía de la artista y de Mitchell-Innes \& Nash, Nueva York; páginas ,78 y 79, 7, 8 y 9. @ Katharina Grosse, VEGAP, Sevilla, 2021; páginas 82 y 83, 10 , 11 y 12. Heather Day website. Disponible en: https://heatherday.com/downtownoakland; página 90, 1. Universidad de Zaragoza. Unidad Técnica de Construcciones.; página 91, 2. Planimetría redibujada por Clara Dobón Ricarte.; página 92, 3. Planos, redibujada por Clara Dobón Ricarte. Fotografías cortesía de la Facultad de Derecho de la Universidad de Zaragoza.; página 93, 4. Google Maps.; página 94, 5. Fotografías de los autores.; página 95, 6. Proyecto de Ejecución, Ampliación y Reforma de la Facultad de Derecho (Zaragoza). Fuente: Universidad de Zaragoza. Unidad Técnica de Construcciones.; páginas 96 y 98, 7, 8. Planos y axonometría dibujados por Clara Dobón Ricarte; páginas 99 y 101, 9, 10, 11. Fotografías de los autores.; página 102, 12. Fotografía (izquierda) de los autores y fotografía (derecha) cortesía de la Facultad de Derecho de la Universidad de Zaragoza.; página 102, 13. Fotografías de los autores.; página 103, 14. Fotografía cortesía de Javier López Sánchez, decano de la Facultad de Derecho de la Universidad de Zaragoza.; página 104, 15. Proyecto de Ejecución, Ampliación y Reforma de la Facultad de Derecho (Zaragoza). Fuente: Universidad de Zaragoza. Unidad Técnica de Construcciones; página 108, 1. Dibujos del autor; página 109, 2. Ayuntamiento de Madrid; página 110 y 112, 3 y 4. Ayuntamiento de Madrid; página 113 y 114, 5 y 6 . Archivo Cabrero; página 115, 7. Reconstitución gráfica del autor; página 116, 8. Legado 2 Fundación COAM; página 117, 9. Dibujos del autor; página 118, 10. Arquitectura, 1959, n. 7 y Francisco de Asís Cabrero y Jaime Ruiz; página 119, 11. Archivo Cabrero; página 120, 12. Dibujos del autor; página 120, 13. Fotografía del autor; página 121, 14. Dibujos del autor; página 122, 15. Fotografía del autor. 

CITY. Darío Álvarez Álvarez • LINAZASORO EN REIMS. EL ESPACIO PÚBLICO COMO MEMORIA DEL LUGAR / LINAZASORO IN REIMS. THE PUBLIC SPACE AS THE MEMORY OF THE PLACE. Victoriano Sainz Gutiérrez • EDITAR VS. CONSTRUIR: UNA ECOLOGÍA DE LO INVISIBLE. AMPLIFICAR LA COMPRENSIÓN DE LAS TÉCNICAS DE PROYECTO / EDITING VS. BUILDING: AN ECOLOGY OF THE INVISIBLE. AMPLIFYING THE UNDERSTANDING OF ARCHITECTURAL DESIGN TECHNIQUES. Paula Victoria Álvarez Benítez • CUANDO LA PINTURA AMPLÍA LA ARQUITECTURA: INTERVENCIONES REALIZADAS EN EL ESPACIO PÚBLICO / WHEN PAINTING ENHANCES ARCHITECTURE: INTERVENTIONS IN A PUBLIC SETTING. Aurora Alcaide-Ramírez; Ana Ruiz-Abellón • UN EDIFICIO INVISIBLE. NUEVO AULARIO DE LA FACULTAD DE DERECHO DE LA UNIVERSIDAD DE ZARAGOZA (1983-1996) / AN INVISIBLE BUILDING. NEW LECTURE ROOM BUILDING OF THE FACULTY OF LAW OF THE UNIVERSITY OF ZARAGOZA (1983-1996). Luis Miguel Lus-Arana; Lucía Carmen Pérez-Moreno • ARQUITECTURAS AMPLIADAS. EL PABELLÓN DE EXPOSICIONES EN LA CASA DE CAMPO DE MADRID) / EXPANDED ARCHITECTURES. THE EXHIBITION PAVILION AT THE CASA DE CAMPO IN MADRID. José de Coca Leicher $\bullet$

- RAFAEL MONEO VALLÉS: LA VIDA DE LOS EDIFICIOS. LA MEZQUITA DE CÓRDOBA, LA LONJA DE SEVILLA Y UN CARMEN EN GRANADA . Víctor Pérez Escolano • FRANCISCO DE GRACIA: CONSTRUIR EN LO CONSTRUIDO. LA ARQUITECTURA COMO MODIFICACIÓN. Pablo Diañez Rubio • FRÉDÉRIC DRUOT, ANNE LACATON \& JEAN-PHILIPPE VASSAL PLUS: LA VIVIENDA COLECTIVA. TERRITORIO DE EXCEPCIÓN. Javier Terrados Cepeda.
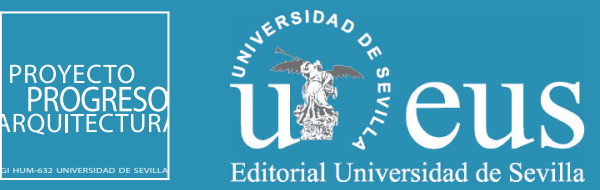